\title{
An anisotropic cohesive phase field model for quasi-brittle fractures in thin fibre-reinforced composites
}

\author{
Udit Pillai $^{\mathrm{a}}$, Savvas Triantafyllou ${ }^{\mathrm{b}, *}$, Yasser Essa $^{\mathrm{c}}$, Federico Martin de la Escalera ${ }^{\mathrm{c}}$ \\ ${ }^{a}$ Faculty of Engineering, The University of Nottingham, University Park, Nottingham NG7 2RD, U.K. \\ ${ }^{b}$ School of Civil Engineering, National Technical University of Athens, 9, Heroon Polytechniou Str., 15780 Zografou, Greece \\ ${ }^{c}$ Aernnova Engineering Division S.A., Avda. del Llano Castellano 13, 28034 Madrid, Spain
}

\begin{abstract}
Thin unidirectional-tape and woven-fabric composites are widely utilized in the aerospace and automotive industries due to their enhanced fatigue life and damage resistance. Providing high-fidelity simulations of intra-laminar damage in such laminates is a challenging task both from a physics and a computational standpoint, due to their complex and largely quasi-brittle fracture response. This is manifested by matrix cracking and fibre breakage, which result in a sudden loss of strength with minimum crack openings; subsequent fibre pull-outs result in a further, although gradual, strength loss. To effectively model this response, it is necessary to account for the cohesive forces evolving within the fracture process zone. Furthermore, the interaction of the failure mechanisms pertinent to both the fibres and the matrix necessitate the definition of anisotropic damage models.

We propose a cohesive phase-field model to simulate intra-laminar fracture in fibre reinforced composites. To capture damage anisotropy, distinct energetic crack driving forces are defined for each pertinent composite damage mode together with a structural tensor that accounts for material orientation dependent fracture properties. The material degradation is governed by a 3-parameter quasi-quadratic degradation function, which can be calibrated to experimentally derived strain softening curves. The proposed damage model is implemented in Abaqus and is validated against experimental results.
\end{abstract}

Keywords: Quasi-brittle fracture, Fibre reinforced composites, Anisotropic cohesive phase-field model, Experimental verification, Finite element simulation

\section{Introduction}

The demand for lighter, stronger, and durable structures has led to the widespread application of fibre-reinforced composites, especially in the aerospace and the automobile industries. Compared to unidirectional composites, traditional fabric-reinforced composites exhibit lower in-plane stiffness and critical strength due to the waviness effect of the fibres $[1,2]$. However, fabric-reinforced composites possess higher through-thickness damage resistance and are capable of dissipating higher energy during critical impact events. These properties render them an ideal solution for improving the crashworthiness of composite structures. Thin uni-directional (UD) tapes and woven-fabric CFRPs manufactured using the spreadtow technology [3] are a special class of composites that have been of significant research interest in the past few years.

In spread tow technology, the fibres are spread into thinner, wider and flatter reinforcements thereby producing ultra lightweight composites with improved stiffness, strength and impact resistance [4]. In particular, spread-tow textile fabrics are known to possess a significantly lower fibre-waviness, reduced resin-rich pockets, lower crimp angles and crimp frequencies [5]. As a result, a higher number of thin plies with

\footnotetext{
${ }^{*}$ Corresponding author

Email address: savtri@mail.ntua.gr (Savvas Triantafyllou )
}

smaller relative fibre angles can be stacked within the same laminate width, resulting in composites with increased toughness against interfacial fracture between the plies [6, 7]. Spread-tow fabrics (STF) provide a similar mechanical performance as that of a cross plied unidirectional ply, but with improved drapability and delamination resistance [8].

Intra-laminar failure in most composites initiates within resin rich matrix pockets, which further evolve to cause delamination, and also fibre failure in some cases. However in thinply STF laminates, fibre-based failures like fibre breakage and pullout are the primary and predominant damage mechanisms, while sub-critical mechanisms like transverse matrix cracking and delamination are delayed or suppressed in most cases, [see, e.g., 9, 7, 10, 11, 4]. Damage modelling in such laminates often becomes cumbersome as fibre-based failures result in a quasi-brittle fracture behaviour. Fibre-based damage mechanisms comprise combined fibre fracture and pullout events that result in the development of a fracture process zone (FPZ) due to fibre-bridging between the crack faces. This gives rise to cohesive fractures associated with a gradual crack growth and a corresponding reduction in the laminate strength; this is in contrast to brittle fractures which are manifested by a sudden drop in the induced stresses and subsequent structural collapse.

In addition, the occurrence of matrix cracks under in-plane shear stress states leads to complex anisotropic fracture phenomena accompanied by a wide variety of interacting damage 
mechanisms, e.g., fibre breakage/pullout under in-plane tension, fibre kink band formation under in-plane compression, matrix cracking under in-plane tension/shear, and fibre-matrix debonding [7]. This necessitates the need for robust damage modelling algorithms which are capable of accurately predicting both critical fracture strengths as well as anisotropic crackpatterns, thus providing a reliable assessment of the overall structural integrity.

The accurate simulation of evolving crack paths in thin-ply laminates is an arduous and challenging task due to the presence of such significantly diverse and coupled intra-laminar failure modes. Several theories and criteria have been proposed in the past to predict intra-laminar damage in composite laminates $[12,13,2,14,15,16,17]$. The most popular among these are the constitutive models based on Continuum Damage Mechanics (CDM) [18, 19, 20], which have reached a mature development state and are the industry standard. CDM represents defects and cracks in the form of a homogeneous continuum damage field distributed over the entire macroscopic solid domain; the material degradation effects are accounted by an appropriately defined damage variable. CDM was first applied for damage prediction in composites by Talreja [21, 22] who also later studied damage evolution under fatigue loading [23]. Prominent work was done by Ladeveze and LeDantec [24] (see, also, [25]) in developing a CDM based damage model for UD composites which was also later extended to fabric-reinforced composites [26, 27, 28]. Böhm et al. [29] developed a phenomenological damage model to describe quasi-brittle fracture behaviour in textile composites with non-crimp reinforcement.

To this point, considerable research has been performed for the development of CDM-based models for UD [12, 13] and thin woven fabric-reinforced composites [30, 2], which have also been applied to simulate impact damage problems $[31,4,32]$. However, when strain softening behaviour is included within the CDM framework the corresponding boundary value problem becomes ill-posed often resulting in numerical instabilities [12, 33, 34]. Consequently, the finite-element solution becomes mesh-dependent and additional energy-based regularization techniques, e.g., the crack-band model, must be employed to prevent spurious damage localization and ensure an admissible mesh-objective solution [35, 13].

The phase-field method (PFM), originated from the works of Francfort and Marigo [36], Bourdin et al. [37], is a relatively recent but robust method for the simulation of evolving cracks in deformable media. The primary advantage of PFM lies in its capability of automatically predicting mesh-independent curvilinear crack patterns including branching and merging; this motivates its application to the simulation of complex intra-laminar composite failure mechanisms. The current applications of PFM include brittle fractures [38, 39, 40, 41, 42, 43], quasibrittle fractures [44, 45], ductile fractures [46, 47, 48, 49], hydraulic fractures [50, 51], and hydrogen assisted fractures [52]. PFM has also been implemented in the commercial software Abaqus [53, 54, 55, 56] within user-element (UEL) and usermaterial (UMAT) subroutines.

With regards to composite applications, Verhoosel and Borst [57], (see also, [58]) attempted to model inter-laminar failures in composite laminates by incorporating the cohesive zone approach in a variational phase-field framework. Despite the significant advantages offered by the PFM, there have been only limited applications vis-à-vis the simulation of composite intralaminar fracture problems. Although there have been a wide variety of anisotropic phase-field models developed till-date, see e.g., $[59,60,61]$, most of them are based on a unique fracture toughness distribution defined with respect to crack orientation. This is not in line with the actual fracture behaviour of composites, wherein each intra-laminar failure mode, i.e., fibre breakage under tension, matrix cracking under tension/shear, have different critical energy release rates associated with it [14]. Moreover, most currently available anisotropic phasefield models assume material properties to be isotropic which is in contrast to composites which display highly anisotropic material behaviour corresponding to matrix and fibre directions.

Bleyer and Alessi [62] modelled different composite damage mechanisms considering distinct phase-fields for each individual damage mechanism. Reinoso et al. [63] predicted the strength of notched thin-ply laminates using a homogeneous isotropic phase-field model and compared it to a finite fracture mechanics (FFM) approach. Dean et al. [64] coupled the phase-field method with a pressure-dependent phenomenological elasto-plastic material model for ductile fracture in short fibre reinforced composites. Natarajan et al. [65] studied the role of fiber-orientations and the inter-fiber spacing on the fracture characteristics of constant/variable stiffness composite laminates using a brittle phase-field model. Reinoso et al. [66] and Guillén-Hernández et al. [67] combined phase field and cohesive zone models to investigate failure initiation at composite micro-scales. Significant efforts have been made to model the interaction between intra-laminar and inter-laminar failures in composites by coupling the brittle phase-field model and cohesive zone method in a physically consistent manner, see e.g., [68, 69, 70]. Recently, Quintanas-Corominas et al. [14] developed a novel and robust strategy to simulate intra-laminar and trans-laminar brittle fracture in long-fibre composites by incorporating anisotropy in both the elastic and fracture properties. Their implementation relied on an additive split of the crackdriving forces associated with each individual damage mechanism. The same phase-field variable was associated with all driving forces. However, it was also highlighted that this approach results in a single stress-degradation function that triggers the degradation of all elastic properties simultaneously and by the same amount, which eventually may lead to premature triggering of failure in a full laminate analysis.

In the current work, we introduce a novel anisotropic cohesive phase field formulation that can accurately model the quasi-brittle failure behaviour exhibited by most thin UD and woven-fabric composite laminates. Motivated by the work of Lorentz [71] on non-local gradient damage modelling for cohesive fractures in plain concrete we introduce a single phasefield variable to describe damage in composites. However, to account for the different damage mechanisms of a composite material, in our formulation the stresses and elastic properties in each material orientation are degraded using distinct degradation functions that depend on the critical energy release rate 
and the fracture stress along that direction. The crack driving state functions and stress-degradation functions corresponding to each individual damage mechanism, e.g., in-plane longitudinal/transverse cracks in the fibres and shear cracks in the matrix, are determined separately. An anisotropic structural tensor is defined to orient the crack-path in the direction associated with the lower fracture strength and critical energy release rate. Furthermore, we introduce a 3-parameter variant of the cohesive phase-field model that provides higher flexibility in controlling the shape of the cohesive softening law. This is important because the damage models in which the initial and the final stages of the cohesive softening curve cannot be controlled independently have only limited capability in accurately predicting the post-fracture material response [72, 71].

The paper is structured as follows. In Sec. 2, the isotropic cohesive phase-field model proposed in Geelen et al. [45] is briefly revisited. Next, a modified anisotropic 3-parameter cohesive phase-field model is proposed in Sec. 3 to simulate quasi-brittle anisotropic fracture scenarios in thin spread-tow unidirectional and fabric-reinforced composites. Sec. 4 provides a detailed description of our numerical implementation in the commercial FEM software Abaqus. Furthermore, a strategy based on formulations in Lorentz [71] is provided in Sec. 5 , to obtain material softening law by calibrating the values of cohesive-shape parameters with respect to the experimental response. Benchmark numerical examples are presented in Sec. 6 along with experimental validations performed on spread-tow composite laminates, followed by conclusions in Sec. 7 .

\section{Isotropic cohesive phase-field model}

The isotropic cohesive phase-field model proposed in [44, 45] for simulating quasi-brittle fractures is briefly revisited. Within a variational description of cracked solids, the potential energy can be represented as the sum of the elastic strain energy and the fracture energy as shown in Eq. (1).

$$
\Pi(\mathbf{u}, \Gamma)=\int_{\Omega} \psi(\varepsilon(\mathbf{u})) d \Omega+\int_{\Gamma} \mathcal{G}_{c} d \Gamma,
$$

where $\Omega$ is the entire solid domain, $\Gamma$ is the crack-path as shown in Fig. 1, $\varepsilon$ is the linearized strain tensor, $\mathbf{u}$ is the displacement vector at any arbitrary point in $\Omega, \psi$ is the elastic strain energy density, and $\mathcal{G}_{c}$ is the critical energy release rate. The linearized strain tensor $\varepsilon$ is defined according to Eq. (2) as

$$
\varepsilon=\frac{\nabla \mathbf{u}+\nabla^{\mathbf{T}} \mathbf{u}}{2} \text {. }
$$

In the variational phase-field theory [37] the fracture surface energy in Eq. (1), which corresponds to a sharp crack path, is regularized by a diffused crack phase-field represented by a scalar phase-field variable $\phi \in[0,1] ; \phi=1$ corresponds to the fully cracked and $(\phi=0)$ to the fully intact states of the material. The regularized fracture energy is hence expressed according to Eq. (3)

$$
\int_{\Gamma} \mathcal{G}_{c} d \Gamma \approx \int_{\Omega} \mathcal{G}_{c} \gamma(\phi, \nabla \phi) d \Omega,
$$
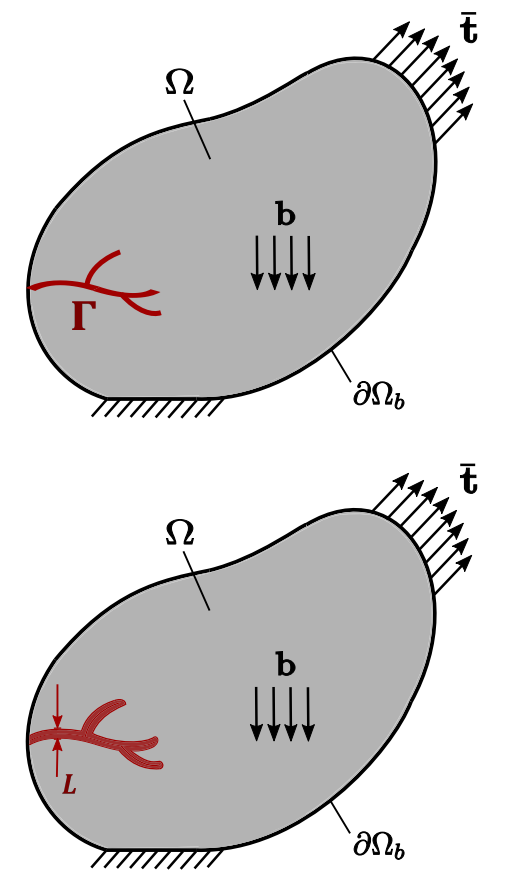

Figure 1: General domain $\Omega$ containing (a) Internal sharp crack $\Gamma$, and (b) Diffused crack with length-scale $L$, and subjected to body force $\mathbf{b}$ and surface traction forces $\overline{\mathbf{t}}$

where, a linear fracture surface energy approximation function $\gamma(\phi, \nabla \phi)$ based on [73] is adopted, with $L$ being the lengthscale parameter that controls the width of the diffusion zone according to Eq. (4).

$$
\gamma(\phi, \nabla \phi)=\frac{3}{4 L}\left[\phi+\frac{L^{2}}{4} \nabla \phi \cdot \nabla \phi\right] .
$$

The linear description of the fracture surface energy in Eq. (4) results in cracks with a finite diffusion width and plays an important role in obtaining a purely elastic material response prior to crack-initiation. This is central to the phase-field description of cohesive fracture [45]. Based on Eqs. (1), (3), and (4) the regularized potential energy of the solid assumes the following form

$$
\begin{aligned}
\Pi(\mathbf{u}, \phi)=\int_{\Omega} \psi(\varepsilon(\mathbf{u}), \phi) d \Omega+\int_{\Omega} \mathcal{G}_{c} \gamma(\phi, \nabla \phi) d \Omega \\
-\int_{\Omega} \mathbf{b} \cdot \mathbf{u} d \Omega-\int_{\partial \Omega_{b}} \overline{\mathbf{t}} \cdot \mathbf{u} d \partial \Omega_{b},
\end{aligned}
$$

where $\mathbf{b}$ and $\overline{\mathbf{t}}$ are externally applied body and traction forces, respectively and $\psi(\varepsilon(\mathbf{u}), \phi)$ is the degraded elastic strain energy density which accounts for the fact that the stored elastic strain energy of the solid must decrease as the crack evolves and the amount of dissipated fracture energy increases.

The elastic strain energy density $\psi(\varepsilon(\mathbf{u}))$ includes contributions from both tensile and compressive deformation modes. However, the crack cannot evolve in regions which are under pure compression. Several methods have been proposed 
to avoid this un-physical behaviour [see, e.g., 38, 39, 74]. The isotropic cohesive phase-field model in [45] follows the approach described in [39] wherein the elastic strain energy $\psi(\varepsilon(\mathbf{u}))$ is split into a tensile and a compressive part; only the active part is responsible for driving the crack evolution process. This is accomplished via a spectral decomposition of the strain tensor into its tensile and compressive components as shown in Eq. (6)

$$
\begin{aligned}
\varepsilon & =\varepsilon^{+}+\varepsilon^{-} \\
\varepsilon^{ \pm} & =\left\langle\lambda_{i}\right\rangle^{ \pm} \mathbf{n}_{\mathbf{i}} \otimes \mathbf{n}_{\mathbf{i}},
\end{aligned}
$$

where the Macaulay brackets imply $\langle x\rangle^{ \pm}=(x \pm|x|) / 2$ and $\lambda_{i}$, $\mathbf{n}_{\mathbf{i}}$ are the eigenvalues and eigenvectors of the strain tensor $\varepsilon$. Based on Eq. (6), the degraded elastic strain energy $\psi(\varepsilon(\mathbf{u}), \phi)$ is additively decomposed into a tensile $\psi^{+}$and a compressive $\psi^{-}$component. To account for the fracture induced material degradation, a degradation function $g(\phi)$ is imposed on the tensile part of the elastic energy density so that

$$
\psi(\varepsilon(\mathbf{u}), \phi)=g(\phi) \psi^{+}\left(\varepsilon^{+}\right)+\psi^{-}\left(\varepsilon^{-}\right),
$$

where

$$
\psi^{ \pm}=\frac{1}{2} \lambda_{s}\left[\langle\operatorname{tr}(\varepsilon)\rangle^{ \pm}\right]^{2}+\mu_{s} \varepsilon^{ \pm}: \varepsilon^{ \pm}
$$

and $\lambda_{s}, \mu_{s}$ are the Lamé constants. Based on the work of Lorentz [71] on an isotropic gradient damage model, the degradation function $g(\phi)$ is defined as

$$
g(\phi)=\frac{(1-\phi)^{2}}{(1-\phi)^{2}+m \phi\left(1+p \phi\left[\exp \left(q^{2} \phi^{2}\right)\right]\right)} ; p \geq 1 .
$$$$
\text { with, ; } m=\frac{3 \mathcal{G}_{c} E}{2 L \sigma_{c}^{2}} ; L<\frac{3 E \mathcal{G}_{c}}{2(p+2) \sigma_{c}^{2}}
$$

In Eq. (9), $E$ is the Young's modulus, $\sigma_{c}$ is the critical fracture stress, and $p$ and $q$ are model parameters. One may refer to [71] for a detailed derivation.

The governing equations of the cohesive phase field model emerge from the minimisation problem

$$
\{\mathbf{u}, \phi\}=\operatorname{Argmin}\{\boldsymbol{\Pi}(\mathbf{u}, \phi)\}
$$

that gives rise to the following coupled strong form

$$
\begin{gathered}
\nabla \cdot \sigma+\mathbf{b}=\mathbf{0} \\
\delta_{\phi} \gamma(\phi, \nabla \phi)=\frac{-g^{\prime}(\phi) \psi^{+}}{\mathcal{G}_{c}}=\tilde{\mathcal{D}}_{\text {iso }} \\
\text { with, } \quad \delta_{\phi} \gamma=\frac{3}{4 L}\left[1-\frac{L^{2}}{2} \nabla \cdot \nabla \phi\right] \text { and } \dot{\phi} \geq 0
\end{gathered}
$$

where $\tilde{\mathcal{D}}_{\text {iso }}$ is the isotropic crack-driving force term and $\sigma$ is the Cauchy stress tensor.

Remark 1. To ensure the stability of the cohesive phase field model, [71] derived an upper bound for the length scale parameter of Eq. (9); this is

$$
L<\frac{3 E G_{c}}{2\left(A^{\prime}(0)+2\right) \sigma_{c}^{2}},
$$

where $A(\phi)=1+p \phi\left[\exp \left(q^{2} \phi^{2}\right)\right][71]$ has been used in Eq. (9).

\section{The anisotropic cohesive phase field model for compos- ites}

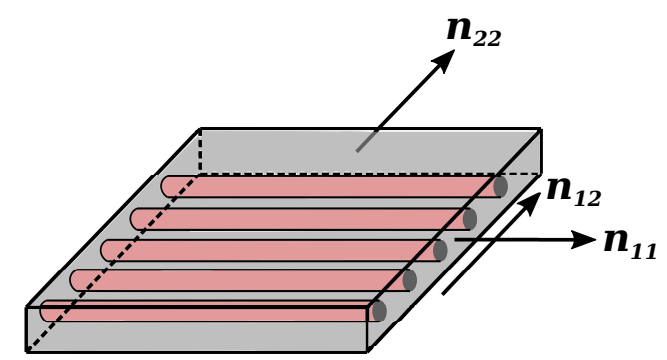

(a)

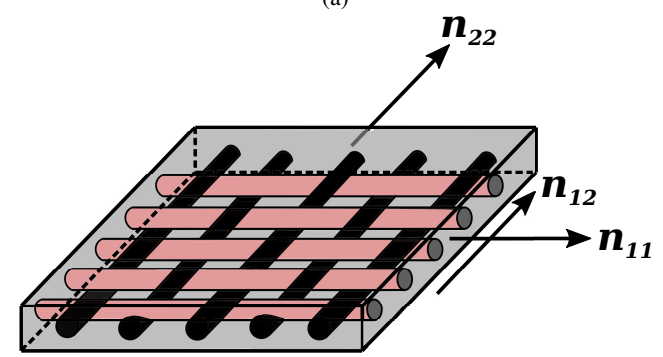

(b)

Figure 2: Illustration of directions $n_{11}, n_{22}$ and $n_{12}$ in thin spread-tow (a) unidirectional and (b) woven-fabric composite plies

Composites are highly anisotropic materials that fail under diverse modes; these can be primarily classified into fibre fracture and pullouts/bridging and matrix rupture. Often, the fracture behaviour displayed by different composite damage mechanisms is quasi-brittle in nature and a fracture-process zone evolves due to the presence of fibre pullout/bridging effects as shown in Fig. 3. Each mechanism not only differs in the driving forces that trigger crack initiation, but they are also associated with different values of the fracture stresses and the corresponding critical energy release rates. This necessitates the extension of the cohesive phase-field model discussed in Sec. 2 to incorporate anisotropic effects, which can accurately capture the fracture response pertaining to each composite damage mechanism.

Driven by the derivations provided in $[75,59]$ and also in $[65,14,76]$ for composite materials, we begin by introducing a structural tensor $\mathcal{A}$ in the definition of the functional $\gamma(\phi, \nabla \phi)$ (see, Eq. (4))

$$
\gamma(\phi, \mathcal{A}, \nabla \phi)=\frac{3}{4 L}\left[\phi+\frac{L^{2}}{4} \nabla \phi \cdot \mathcal{A} \nabla \phi\right] .
$$

The generalised functional definition of Eq. (13) enables the model to drive the crack along the directions with the lower fracture toughness $\sigma_{c}$ and critical energy release rate $\mathcal{G}_{c}$.

In unidirectional composites, the fracture properties in the direction normal to the fibre orientation are substantially weaker when compared to the fibre directions. This implies that it is comparatively much more difficult to crack the fibres and experimental evidence suggests that the crack propagates in the direction parallel to fibres [77]. In thin woven fabric laminates, e.g., in plies with fibre orientations $\left(0^{\circ} / 90^{\circ}\right)$, cracking in either 
direction would involve fibre failure, but the crack might still have a preferred orientation based on the values of fibre zfracture strength and toughness in different directions. Hence, in the current work the structural tensor $\mathcal{A}$ is defined according to Eq. (14) in line with the definition in [14].

$$
\mathcal{A}=\alpha_{1} n_{11} \otimes n_{11}+\alpha_{2} n_{22} \otimes n_{22}
$$

In the case of UD composites, $n_{11}$ and $n_{22}$ correspond to the fibre and matrix directions, respectively (Fig. 2a). In the case of woven-fabric composites, both $n_{11}$ and $n_{22}$ correspond to the fibre directions as shown in Fig. 2b.

Furthermore, $\alpha_{n}=\left\{\alpha_{1}, \alpha_{2}\right\}$ are parameters that penalize crack propagation along the material directions which are manifested with a higher fracture toughness and strength. These parameters assume a value as $\alpha_{n}=1$ along the stronger material direction. Conversely, a very low value is considered for the weaker direction (typically $\alpha_{n} \approx 0.02-0.05$ ). Specific values can also be obtained on the basis of the ratio of critical energy release rates $G_{c(s)}$ and $G_{c(w)}$ along the stronger and weaker directions, respectively, as discussed in $[62,76]$.

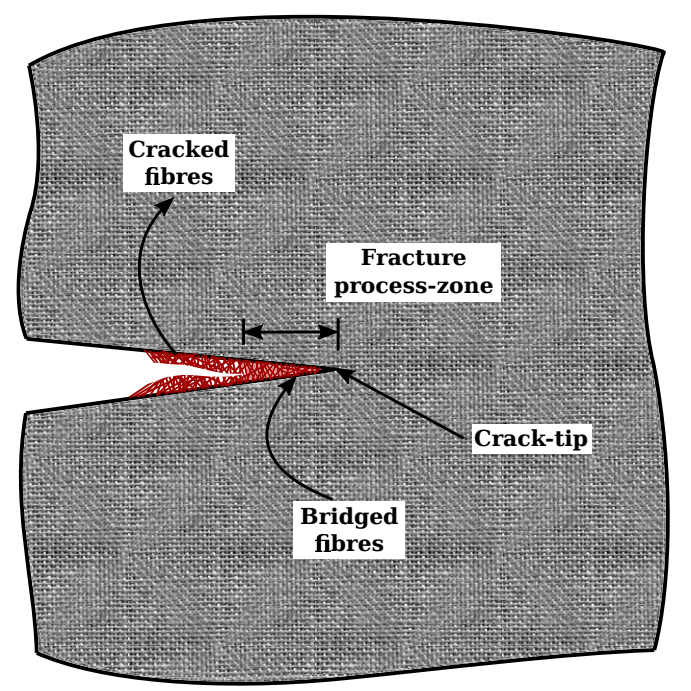

Figure 3: Illustration of fracture-process zone (FPZ) in fibre-reinforced composite plies due to combined fibre breakage and fibre pullout/bridging effect

\subsection{Constitutive assumptions}

The decomposition of the strain energy density $\psi$ and the corresponding crack-driving force $\tilde{\mathcal{D}}_{i s o}$ defined in Eqs. (8) and (11), respectively are valid as long as the material is isotropic in its elastic and post-fracture response. To this point, anisotropic phase field models have been developed, see, e.g. [59, 60, 61] that rely on the assumption of elastic isotropy and consider an anisotropic definition of the critical energy release-rate $\mathcal{G}_{c}$ depending on the crack orientation. However, in composites preferential directions emerge due to the fibre orientations that result in anisotropic elastic properties. This implies that the deformation modes in each direction contribute to specific components of the strain energy density, which then trigger individual failure mechanisms.
Typically, in thin spread-tow fabric and UD plies these failure mechanisms can be classified into the following categories (see, also Fig. 2).

1. Fibre fracture and pullouts in woven-fabric or UD-tape plies due to tensile deformations along the $n_{11}$ direction;

2. In-plane matrix cracking in UD-tape or fibre fracture and pullouts in woven-fabric plies due to tensile deformations along the $n_{22}$ direction, and

3. In-plane matrix cracking in woven-fabric and UD-tape plies, due to shear deformation along the $n_{12}$ direction

The transverse damage mechanisms such as matrix cracking under transverse shear deformations and subsequent delamination are practically suppressed in thin spread-tow composites [7], and hence are not modelled in the current analysis.

In the subsequent sections, the above damage mechanisms are denoted by a subscript $i \in\{11,22,12\}$. The subscripts $\{11\}$, and $\{22\}$ correspond to the tensile failure modes in the material directions $n_{11}$ and $n_{22}$, respectively whereas $n_{12}$ corresponds to the shear failure mode.

Based on the above analysis, it becomes important to individually characterize the active strain energy density components $\psi_{i}$ which are responsible for triggering each corresponding damage mechanism. Similar to [14, 76], we consider that each damage mechanism is associated with a distinct critical energy release rate $\mathcal{G}_{c i}$ and a critical fracture strength $\sigma_{c i}$ that can be experimentally identified.

Within this setting, we reformulate Eq. (11) and introduce the following coupled strong form for our anisotropic cohesive phase-field model

$$
\begin{aligned}
& \nabla \cdot \sigma+\mathbf{b}=\mathbf{0} \\
& \delta_{\phi} \gamma(\phi, \mathcal{A}, \nabla \phi)=\tilde{\mathcal{D}},
\end{aligned}
$$

where

$$
\delta_{\phi} \gamma=\frac{3}{4 L}\left[1-\frac{L^{2}}{2} \nabla \cdot \mathcal{A} \nabla \phi\right] .
$$

The explicit expressions for the Cauchy stress $\sigma$ and the anisotropic crack-driving force $\tilde{\mathcal{D}}$ will be discussed in Sec. 3.2 and Sec. 3.4, respectively.

To ensure that the coupled strong form gives rise to evolving damage only, hence non-healing, the following irreversibility constraint is imposed on the phase field evolution Eq. (16).

$$
\dot{\phi} \geq 0 \text {. }
$$

This irreversibility constraint has been traditionally imposed via a history variable [see, e.g., 39, 41]. In this work, the governing equations are augmented into a Lagrangian setting [45] as discussed in the algorithmic implementation of the proposed model in Section 4.

\subsection{Elastic strain energy density}

Based on the preferential material directions in composites and the classification of different damage mechanisms provided 
in Sec. 3.1, the following split for the strain tensor naturally emerges (see, also Bleyer and Alessi [62])

$$
\varepsilon=\varepsilon^{+}+\varepsilon^{-}
$$

where

$$
\varepsilon^{+}=\left[\begin{array}{c}
\left\langle\varepsilon_{11}\right\rangle^{+} \\
\left\langle\varepsilon_{22}\right\rangle^{+} \\
\gamma_{12}
\end{array}\right]
$$

is the active part and

$$
\varepsilon^{-}=\left[\begin{array}{c}
\left\langle\varepsilon_{11}\right\rangle^{-} \\
\left\langle\varepsilon_{22}\right\rangle^{-} \\
0
\end{array}\right]
$$

is the passive part, respectively. In Eqs. (20) and (21), $\langle x\rangle_{ \pm}=$ $(x \pm|x|) / 2$ are the Macaulay brackets.

Remark 2. It is of interest to note that when using the straindecomposition method shown in Eqs. (20) and (21), the orthogonality condition for the positive/negative stresses is not necessarily satisfied, i.e. the cross-components $\left(\sigma_{i j}^{+} \cdot \varepsilon_{i j}^{-}\right)$in the original strain energy density expression do not necessarily vanish. This aspect has been addressed in the recent work of Wu et al. [78] wherein a positive/negative projection in the energy norm is employed leading to a well-defined energy functional and a thermodynamically consistent framework.

Despite this theoretical inconsistency, we find the split presented in [62] to provide an intuitive framework vis-a-vis the failure response of composites since the distinct failure mechanisms are primarily driven by the prevalent components of the energy density. The extensive experimental validations performed as part of this study (see, also, Section 6) demonstrate that such an assumption is valid and of practical use for the case of composites. Further extending the method provided in Wu et al. [78] for the case of anisotropic fractures would also lend the methodology described herein to a theoretically consistent framework. This is however beyond the scope of the present work.

The effective stress tensor $\bar{\sigma}$ at each material point within each ply is defined accordingly based on Classical Laminate Theory (CLT) as Eq. (22).

$$
\bar{\sigma}=\mathbf{C}_{\mathbf{o}}: \varepsilon
$$

$$
\mathbf{C}_{o}=\left[\begin{array}{ccc}
\frac{E_{11}}{1-v_{12} v_{21}} & \frac{v_{12} E_{22}}{1-v_{12} v_{21}} & 0 \\
\frac{v_{21} E_{11}}{1-v_{12} v_{21}} & \frac{E_{22}}{1-v_{12} v_{21}} & 0 \\
0 & 0 & G_{12}
\end{array}\right] \text { and, } \varepsilon=\left[\begin{array}{l}
\varepsilon_{11} \\
\varepsilon_{22} \\
\gamma_{12}
\end{array}\right],
$$

where $\mathbf{C}_{o}$ is the elastic material stiffness matrix; plane-stress conditions are considered. In Eq. (23), $E_{11}$ and $E_{22}$ are the elastic Young's moduli, $G_{12}$ is the in-plane shear modulus, and $v_{12} / v_{21}$ are the in-plane Poisson's ratios defined for each ply of the composite laminate, where the relation $v_{21} E_{11}=v_{12} E_{22}$ holds.
The effective stress $\bar{\sigma}$ in Eq. (22) can be similarly split into an active and passive part, i.e.,

$$
\bar{\sigma}=\bar{\sigma}^{+}+\bar{\sigma}^{-}
$$

with

$$
\bar{\sigma}^{+}=\left[\begin{array}{c}
\left\langle\bar{\sigma}_{11}\right\rangle^{+} \\
\left\langle\bar{\sigma}_{22}\right\rangle^{+} \\
\bar{\tau}_{12}
\end{array}\right]
$$

and

$$
\bar{\sigma}^{-}=\left[\begin{array}{c}
\left\langle\bar{\sigma}_{11}\right\rangle^{-} \\
\left\langle\bar{\sigma}_{22}\right\rangle^{-} \\
0
\end{array}\right],
$$

respectively.

Based on Eqs. (19)-(21) and (24)-(26), the components of the elastic strain energy density $\psi$, which are responsible for driving the different composite failure mechanisms emerge as

$$
\begin{aligned}
\psi_{11}^{+} & =\frac{1}{2}\langle\bar{\sigma}\rangle_{11}^{+}\langle\varepsilon\rangle_{11}^{+} & \psi_{11}^{-} & =\frac{1}{2}\langle\bar{\sigma}\rangle_{11}^{-}\langle\varepsilon\rangle_{11}^{-} \\
\psi_{22}^{+} & =\frac{1}{2}\langle\bar{\sigma}\rangle_{22}^{+}\langle\varepsilon\rangle_{22}^{+} & \psi_{22}^{-} & =\frac{1}{2}\langle\bar{\sigma}\rangle_{22}^{-}\langle\varepsilon\rangle_{22}^{-} \\
\psi_{12}^{+} & =\frac{1}{2} \bar{\tau}_{12} \gamma_{12} & \psi_{12}^{-} & =0 .
\end{aligned}
$$

In this work, each active energy term $\psi_{11}^{+}, \psi_{22}^{+}, \psi_{12}^{+}$contributes to a specific damage mechanisms, i.e., $\{11\},\{22\}$, and $\{12\}$, respectively and are hence accounted for separately. It is of interest to note that, the isotropic formulations can be recovered by summing up the corresponding active and passive components of strain energy density, i.e.,

$$
\begin{aligned}
& \psi^{+}=\psi_{11}^{+}+\psi_{22}^{+}+\psi_{12}^{+} \\
& \psi^{-}=\psi_{11}^{-}+\psi_{22}^{-}+\psi_{12}^{-} .
\end{aligned}
$$

\subsection{Energetic degradation function}

In this section, we provide explicit definitions of degradation functions pertaining to each composite damage mechanism. Each function is responsible for degrading specific strain energy components and can aid in avoiding premature initiation of failure in all modes simultaneously. Following the derivations presented in [71] (see, also Eq. (9)) for the case of an isotropic medium, a 3-parameter quasi-quadratic degradation function $g_{i}(\phi)$ is introduced for each anisotropic damage mechanism $i \in\{11,22,12\}$, which depends on the critical fracture stress, critical energy release rate and the cohesive law shape corresponding to that mechanism. The degradation functions $g_{11}(\phi), g_{22}(\phi)$ and $g_{12}(\phi)$ are expressed as

$$
\begin{aligned}
& g_{11}(\phi)=\frac{(1-\phi)^{2}}{(1-\phi)^{2}+m_{11} \phi A_{11}(\phi)} \\
& g_{22}(\phi)=\frac{(1-\phi)^{2}}{(1-\phi)^{2}+m_{22} \phi A_{22}(\phi)} \\
& g_{12}(\phi)=\frac{(1-\phi)^{2}}{(1-\phi)^{2}+m_{12} \phi A_{12}(\phi)},
\end{aligned}
$$


where the parameters $m_{11}=-g_{11}^{\prime}(0), m_{22}=-g_{22}^{\prime}(0)$ and $m_{12}=-g_{12}^{\prime}(0)$ correspond to the initial slope of the corresponding degradation functions and are defined as

$$
\begin{aligned}
& m_{11}=\frac{3 \mathcal{G}_{c 11} E_{11}}{2 L \sigma_{c 11}^{2}} \\
& m_{22}=\frac{3 \mathcal{G}_{c 22} E_{22}}{2 L \sigma_{c 22}^{2}} \\
& m_{12}=\frac{3 \mathcal{G}_{c 12} G_{12}}{2 L \sigma_{c 12}^{2}}
\end{aligned}
$$

and $L$ is a length scale. Furthermore $A_{11}, A_{22}, A_{12}$ assume the following form

$$
\begin{array}{r}
A_{11}(\phi)=1+p_{11} \phi\left[\exp \left(q_{11}^{2} \phi^{2}\right)\right] \\
A_{22}(\phi)=1+p_{22} \phi\left[\exp \left(q_{22}^{2} \phi^{2}\right)\right] \\
A_{12}(\phi)=1+p_{12} \phi\left[\exp \left(q_{12}^{2} \phi^{2}\right)\right],
\end{array}
$$

where $q_{i}$, and $p_{i} \geq 1$ are model parameters.

The degradation functions $g_{i}(\phi)$ defined in Eqs. (30)-(32) are imposed on the active tensile strain energy densities $\psi_{i}^{+}$in Eq. (27), hence resulting in the following equation

$$
\psi=g_{11} \psi_{11}^{+}+g_{22} \psi_{22}^{+}+g_{12} \psi_{12}^{+}+\psi_{11}^{-}+\psi_{22}^{-}+\psi_{12}^{-} .
$$

Hence, contrary to the isotropic case of Eq. (7), in the anisotropic case each failure mode specific degradation function $g_{i}(\phi)$ acts only on its respective active energy term $\psi_{i}^{+}$. This ensures that once the damage is triggered, the material compliance in each direction is penalised by different amounts of degradation based on mode-specific elastic and fracture properties.

Remark 3. The 3-parameter degradation function chosen in this work allows for a versatile and accurate simulation of damage evolution in composites. Cohesive laws in which the initial and the final stages of softening cannot be controlled independently have been shown to offer limited capabilities in highfidelity damage simulations [72, 71]. The model can be further extended by introducing additional parameters and higherorder polynomials in the degradation function definition, see e.g., [44], to obtain more diverse softening behaviour.

The model parameters $\left\{m_{i}, p_{i}, q_{i}\right\}$ control the shape of the cohesive softening law for each individual failure mode $i \in$ $\{11,22,12\}$. Their effect on the degradation functions $g_{i}(\phi)$ are shown in Fig. 4. In particular, parameter $p_{i}$ controls the initial slope of the degradation function only (Fig. 4a). Higher values of $p_{i}$ result in faster material degradation hence reducing the predicted peak fracture stresses. Conversely, $q_{i}$ controls the final stage of the degradation function only (Fig. 4b). Thus, it affects the final crack-separation displacement in the cohesivelaw.

The third parameter $m_{i}$, see Eq. (33)-(35), depends on the material and fracture properties $\left(E_{i}, \sigma_{c i}, \mathcal{G}_{c i}\right)$ which are constants for any given material and affect the shape of the softening branch. Smaller values give rise to near-linear descending branches (Fig. 4c). The fact that $m_{i}$ depends also on the length scale $L$, which is common for all failure modes, implies that $\left\{m_{11}, m_{22}, m_{12}\right\}$ cannot be calibrated independently of each other. However, as the fracture response of cohesive phasefield models has been shown to be length scale independent, $L$ needs only to assume a small enough value so that the diffusion zone becomes bounded [44, 45]. In the limit $L \rightarrow 0$, the cohesive phase-field model converges to an asymptotic cohesive zone model, as demonstrated in [71]. It is worth noting that in the current model, the estimated peak fracture force and the final crack-separation displacement remain unaffected due to variations of parameter $m_{i}$ and the length-scale $L$.

Furthermore, it can be observed that due to the specific formulations adopted for $g_{i}(\phi)$ in Eq. (30), the stresses in each direction are naturally subjected to different amounts of degradation, which is controlled by the anisotropic fracture parameters $\left\{\mathcal{G}_{c i}, \sigma_{c i}\right\}$; these correspond to specific composite failure modes $i \in\{11,22,12\}$.

Remark 4. Similar to Eq. (12), an equivalent upper bound definition for the length-scale must be incorporated within the proposed anisotropic cohesive phase-field model to achieve optimal convergence. We define this based on the material and fracture properties in two principal, viz. warp and weft, directions of the spread-tow fabric and spread-tow unidirectional composites, i.e.,

$$
L<L_{(U B)}=\min \left(\frac{3 E_{11} \mathcal{G}_{c 11}}{2\left(A_{1}^{\prime}(0)+2\right) \sigma_{c 11}^{2}}, \frac{3 E_{22} \mathcal{G}_{c 22}}{2\left(A_{2}^{\prime}(0)+2\right) \sigma_{c 22}^{2}}\right),
$$

where $\mathcal{G}_{c i}$ are the critical energy release rates, $\sigma_{c i}$ are the critical fracture stresses and $L$ is the length scale parameter with its upper-bound defined by $L_{(U B)}$. The expression in Eq. (40) is closely related to the characteristic length of the fracture process zone, which is given as $L_{F P Z_{i}}=E_{i} \mathcal{G}_{c i} / \sigma_{c i}^{2}$.

\subsection{Anisotropic crack-driving force}

The definition of failure mode specific degradation functions ensures that these degrade only the corresponding active part of the elastic energy density Eq. (28), with the degradation rates governed by respective material and fracture properties. This ascertains that despite using a single damage variable in our formulations, we maintain a slower damage evolution rate in stronger material directions post damage initiation. This anisotropic degradation effect must also be manifested within the definitions of the crack-driving forces responsible for triggering each failure mode. To ensure independent crack evolution in each direction, (see Fig. 5 for a woven-fabric composite ply), we consider that the crack-driving force $\tilde{\mathcal{D}}$ in Eq. (16) is additively decomposed into failure mode specific components, i.e.,

$$
\tilde{\mathcal{D}}=\tilde{\mathcal{D}}_{11}+\tilde{\mathcal{D}}_{22}+\tilde{\mathcal{D}}_{12}
$$

where

$$
\begin{aligned}
& \tilde{\mathcal{D}}_{11}=-\frac{g_{11}^{\prime} \psi_{11}^{+}}{\mathcal{G}_{c 11}}, \\
& \tilde{\mathcal{D}}_{22}=-\frac{g_{22}^{\prime} \psi_{22}^{+}}{\mathcal{G}_{c 22}},
\end{aligned}
$$




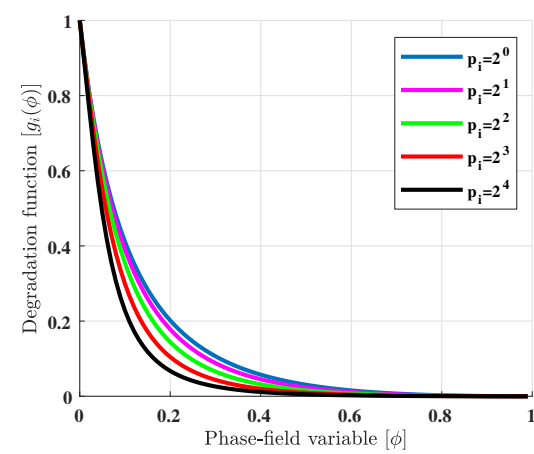

(a)

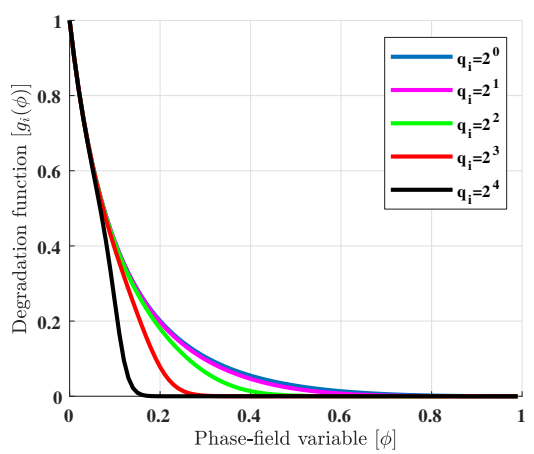

(b)

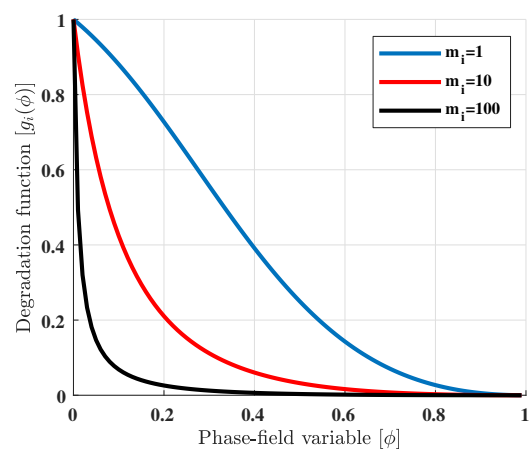

(c)

Figure 4: Variation of stiffness degradation function $g_{i}(\phi)$ for any composite damage mechanism $i$ with respect to phase-field variable $\phi$ for different values of cohesive shape parameters $\left\{m_{i}, p_{i}, q_{i}\right\}$

and

$$
\tilde{\mathcal{D}}_{12}=-\frac{g_{12}^{\prime} \psi_{12}^{+}}{\mathcal{G}_{c 12}},
$$

respectively.

In Eqs. (42)-(44), the mode specific crack driving force terms depend on the mode specific critical energy release rates $\mathcal{G}_{c i}$, the tensile strain energy densities $\psi_{i}^{+}$, and the degradation functions $g_{i}(\phi)$. Hence, the coupling between the governing Eqs. (15) and (16) is indeed achieved in a failure mode-wise manner.

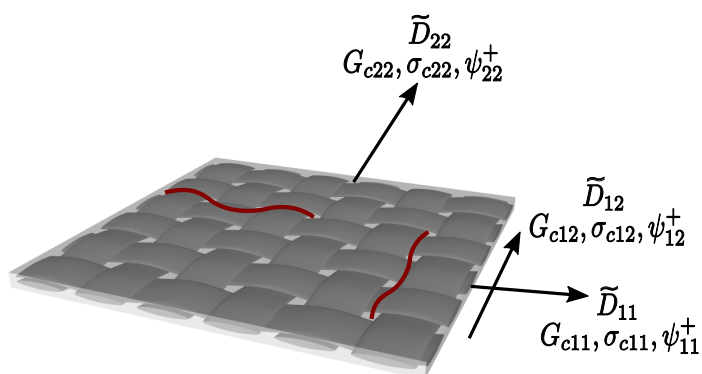

Figure 5: Illustration of different crack-driving forces $\tilde{\mathcal{D}}_{i}$, critical energy release rates $\mathcal{G}_{c i}$, critical fracture stresses $\sigma_{c i}$ and strain energy density contribution $\psi_{i}^{+}$, responsible for initiating each damage mechanism $i$ in spread-tow fabric composites. Each $\tilde{\mathcal{D}}_{i}$ is degraded using distinct degradation function $g_{i}(\phi)$

Remark 5. The isotropic case can be recovered by setting,

$$
\begin{aligned}
& \sigma_{c}=\sigma_{c 11}=\sigma_{c 22}=\sigma_{c 12} \\
& \mathcal{G}_{c}=\mathcal{G}_{c 11}=\mathcal{G}_{c 22}=\mathcal{G}_{c 12}
\end{aligned}
$$

which also eventually leads to,

$$
g(\phi)=g_{11}(\phi)=g_{22}(\phi)=g_{12}(\phi)
$$

\subsection{Effective critical energy release-rate}

Bourdin et al. [37] in their original variational formulation showed that the fracture energy is slightly overestimated during phase-field simulations. The amplitude of this overestimation depends on the characteristic mesh size in the overall finiteelement discretization. To alleviate this effect, the critical energy release rate obtained from the experiments must be scaled to an effective $\mathcal{G}_{c}^{e f f}$ for the purpose of phase-field simulations, see also Egger et al. [79] for a comparative review. In this work, we closely follow the scaling procedure provided in [14]

$$
\mathcal{G}_{c}^{e f f}=\mathcal{G}_{c}\left(1-\frac{h_{e}}{L}\right)
$$

where $h_{e}$ is the characteristic element length, $L$ is the lengthscale parameter, and $\mathcal{G}_{c} / \mathcal{G}_{c}^{e f f}$ are the actual and effective critical energy release rates, respectively. For an effective comparison between the experimental results and the fracture estimations provided by phase-field simulations, it is important to use $\mathcal{G}_{c}^{e f f}$ in the formulations; this is discussed in the numerical examples presented in this study.

\subsection{Shear isotropic hardening in fabric-reinforced composites}

In woven-fabric reinforced composites, the shear response is primarily dominated by the material properties of the matrix. This involves nonlinear plastic deformations of the matrix followed by strength degradation due to the initiation of matrix micro-cracks $[2,4]$. To account for the plasticity and subsequent strength reduction, we combine the anisotropic cohesive phase field model with an elastic-plastic constitutive model with isotropic hardening for the in-plane shear behaviour. The plastic evolution of shear stresses is controlled using a threshold function proposed in [2], also shown in Eq. (48), which governs the onset and evolution of plastic shear behaviour.

$$
\bar{\tau}_{12}=S_{L P}+\zeta_{E}\left[1-e^{-\left|\zeta_{E}^{T} \varepsilon_{12}^{p}\right|}\right]+\zeta_{L} \varepsilon_{12}^{p},
$$

where $\bar{\tau}_{12}$ is the in-plane shear stress, $\varepsilon_{12}^{p}$ is the plastic shear strain, and $S_{L P}$ is the shear yield stress. The constants $\left\{\zeta_{E}, \zeta_{E}^{T}, \zeta_{L}\right\}$ are material parameters that can be obtained through calibration to experimental results obtained from pure shear tests of woven fabric-reinforced composites, see, e.g. [4]. Fig. 6 schematically illustrates the shear stress-strain response for any typical fabric-reinforced composite, based on the elastic-plastic cohesive phase-field model proposed in this work.

The corresponding plastic strain evolution law is given by Eq. (49)

$$
\dot{\varepsilon}_{12}^{p}=\dot{\varepsilon}_{12}^{i} \operatorname{sign}\left(\varepsilon_{12}^{e}\right),
$$


where $\varepsilon_{12}^{e}$ is the elastic shear strain and $\varepsilon_{12}^{i}$ is the isotropic hardening variable [2].

Based on this, the active shear strain energy density $\psi_{12}^{+}$in Eq. (27) and (44) must be modified for the case of fabricreinforced composites, to include only the elastic part of the shear strains as shown in Eq. (50).

$$
\psi_{12}^{+}=\frac{1}{2} \bar{\tau}_{12} \gamma_{12}^{e}
$$

where $\gamma_{12}^{e}$ is the engineering elastic shear strain given by Eq. (51).

$$
\begin{aligned}
& \gamma_{12}^{e}=2 \varepsilon_{12}^{e} \\
& \varepsilon_{12}^{e}=\varepsilon_{12}-\varepsilon_{12}^{p}
\end{aligned}
$$

Once the critical shear stress $S_{L}$ is reached, the post-peak shear stress-degradation is controlled using the cohesive phasefield model with appropriate softening parameters.

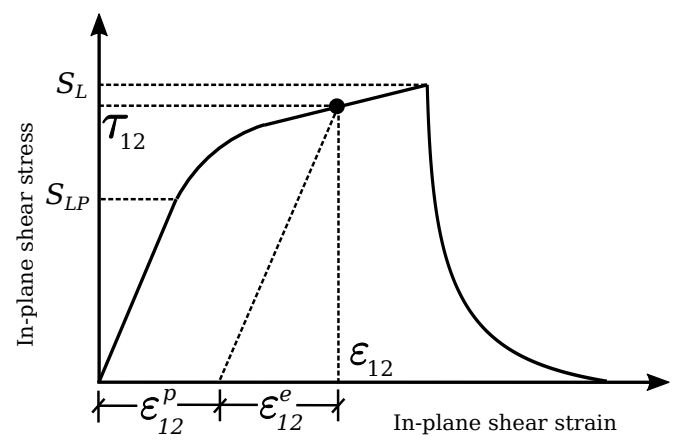

Figure 6: Illustration of characteristic in-plane shear behaviour for a typical spread-tow fabric (STF) reinforced composite

\section{Solution scheme and Abaqus implementation}

\subsection{Augmented Lagrangian form of the governing equations}

Eq. (18) imposes the necessary irreversibility constraint to the phase field, i.e., a crack should not be allowed to heal. Similar to [45], we introduce this constraint by means of an augmented Lagrangian expression and Eq. (16) is re-written as

$\frac{3}{4 L}\left[1-\frac{L^{2}}{2} \nabla \cdot \mathcal{A} \nabla \phi\right]-\left\langle\lambda+\gamma\left(\phi_{n-1}-\phi\right)\right\rangle_{+}-\langle\lambda+\gamma(1-\phi)\rangle_{-}=\tilde{\mathcal{D}}$

where $\lambda$ and $\gamma$ are augmented Lagrange and penalty parameters respectively, and $\left\{\phi_{n-1}\right\}$ is the phase-field variable values obtained at time $t=t_{n-1}$. The penalty $\gamma$ is imposed whenever the value of the phase-field decreases during subsequent time-increments i.e. $\phi<\phi_{n-1}$, or when it exceeds unity i.e. $\phi>1$. The Lagrange multiplier is updated iteratively until convergence. Although this augmented Lagrangian representation minimally distorts the original strong form due the iterative updating of the Lagrange parameter, more accurate values of $\gamma$ can be obtained based on the lower-bound analytical limit presented in [80]. Our numerical experiments have also confirmed the recommended values of $\gamma=1 . e 4$ and $\mathcal{R}_{\gamma}=1.0 e^{-4}$ for the penalty parameter and the corresponding convergence tolerance provide robust and accurate predictions.

\subsection{Galerkin approximation}

To obtain the displacement and phase-field solutions from the equilibrium Eq. (15) $)_{1}$ and the modified phase-field evolution Eq. (52) using the Galerkin method, the following trial solution spaces can be defined.

$$
\begin{aligned}
& \mathcal{S}_{\mathbf{u}}=\left\{\mathbf{u} \in\left(\mathcal{H}^{\mathbf{1}}(\mathbf{\Omega})\right)^{\mathbf{d}} \mid \mathbf{u}=\overline{\mathbf{u}} \text { on } \partial \mathbf{\Omega}_{\mathbf{b}}\right\} \\
& \mathcal{S}_{\phi}=\left\{\phi \in\left(\mathcal{H}^{1}(\Omega)\right)\right\}
\end{aligned}
$$

with the corresponding weighting or test function spaces as defined in Eq. (54).

$$
\begin{aligned}
& \mathcal{W}_{\mathbf{u}}=\left\{\delta \mathbf{u} \in\left(\mathcal{H}^{\mathbf{1}}(\mathbf{\Omega})\right)^{\mathbf{d}} \mid \delta \mathbf{u}=\overline{\delta \mathbf{u}} \text { on } \partial \mathbf{\Omega}_{\mathbf{b}}\right\} \\
& \mathcal{W}_{\phi}=\left\{\delta \phi \in\left(\mathcal{H}^{1}(\Omega)\right)\right\}
\end{aligned}
$$

Multiplying the strong form equations (15) with the above test functions $\delta \mathbf{u}$ and $\delta \phi$, and performing integration by parts leads to the following weak form of governing equilibrium equations (55).

$$
\begin{aligned}
& \mathcal{R}_{\mathbf{u}}=\int_{\Omega} \sigma \cdot \nabla \delta \mathbf{u} \mathbf{d} \boldsymbol{\Omega}-\int_{\mathbf{\Omega}} \mathbf{b} \cdot \delta \mathbf{u} \mathbf{d} \mathbf{\Omega}-\int_{\partial \mathbf{\Omega}_{\mathbf{b}}} \mathbf{t} \cdot \delta \mathbf{u} \mathbf{d} \partial \mathbf{\Omega}_{\mathbf{b}} \approx \mathbf{0} \\
& \mathcal{R}_{\phi}=-\int_{\Omega} \tilde{\mathcal{D}} \delta \phi d \Omega+\int_{\Omega} \frac{3}{4 L}\left[\delta \phi+\frac{L^{2}}{2} \nabla \delta \phi \cdot \mathcal{A} \nabla \phi\right] d \Omega \\
& -\int_{\Omega}\left(\left\langle\lambda+\gamma\left(\phi_{n-1}-\phi\right)\right\rangle_{+}+\langle\lambda+\gamma(1-\phi)\rangle_{-}\right) \delta \phi d \Omega \approx 0
\end{aligned}
$$

The solution to the combined set of Eqs. (55) is obtained using either monolithic or staggered algorithms which are based on simultaneous and alternating minimization respectively, of residuals $\mathcal{R}_{\mathbf{u}}$ and $\mathcal{R}_{\phi}$. Although the conventional monolithic algorithm provides accurate fracture predictions, it suffers from poor convergence issues due to the non-convexity of the underlying energy functional [81]. This has been very recently alleviated by employing the more efficient BFGS monolithic algorithm [82, 83], which involves fewer iterations and reformulations of the system matrix in each increment. In contrast to monolithic approaches, the staggered (alternating minimisation) algorithm relies on decoupling the linear momentum and phase-field equations and solving them separately within each increment. This is achieved by freezing one solution variable at a constant value and solving for another variable, and viceversa. This retains the convexity of the energy functional and hence, displays excellent convergence characteristics.

In the current work, we use a one-pass staggered (alternating minimisation) solution scheme presented in [84], see also [45]. Although such an implementation necessitates the use of small time increments $\left(1.0 e^{-5}-5.0 e^{-5}\right)$ to ensure accuracy, it allows for a straightforward utilization of Abaqus parallel solving capabilities and facilitates the direct visualization of the solution results in Abaqus/Viewer. 


\subsection{Hybrid phase-field formulation}

To accelerate the solution procedure we employ a hybrid formulation, in which the stress and the phase-fields are derived via distinct energy functionals, i.e., $\bar{\psi}$ and $\psi$, respectively. Such a hybrid split based on separate energy functionals $\bar{\psi}$ and $\psi$ has been investigated previously for brittle fractures in [85, 86, 74], quasi-brittle fractures in $[44,87,88,89,90]$ and recently for composite brittle fractures in [76].

According to this, the crack-driving force term $\tilde{\mathcal{D}}$ in Eq. $(55)_{2}$ is evaluated based on the active (tensile) strain energy density expressions $\psi_{i}^{+}$provided in Eq. (41). However, the Cauchy stress in Eq. $(55)_{1}$ is evaluated by the energy functional $\bar{\psi}$ in Eq. (56) below.

$$
\bar{\psi}=\varepsilon^{\mathbf{T}}: \mathbf{C}_{\mathbf{0}}: \varepsilon
$$

where $\mathbf{C}_{o}$ and $\varepsilon$ are defined in Eq. (23). The derivative of $\bar{\psi}$ with respect to $\varepsilon$ provides the effective stresses $\bar{\sigma}$ in Eq. (57), which is similar to the definition shown in Eq. (22)

$$
\bar{\sigma}=\frac{\partial \bar{\psi}}{\partial \varepsilon}=\left[\begin{array}{lll}
\bar{\sigma}_{11} & \bar{\sigma}_{22} & \bar{\tau}_{12}
\end{array}\right]^{\mathbf{T}}=\mathbf{C}_{\mathbf{o}}: \varepsilon
$$

Furthermore, the degraded $(\sigma)$ Cauchy stress tensor can be obtained as shown in Eq. (58) below.

$$
\sigma=\left[\begin{array}{c}
\sigma_{11} \\
\sigma_{22} \\
\tau_{12}
\end{array}\right]=\left[\begin{array}{ccc}
g_{11}(\phi) & 0 & 0 \\
0 & g_{22}(\phi) & 0 \\
0 & 0 & g_{12}(\phi)
\end{array}\right]\left[\begin{array}{c}
\bar{\sigma}_{11} \\
\bar{\sigma}_{22} \\
\bar{\tau}_{12}
\end{array}\right]
$$

The hybrid split conceptually resembles gradient-enhanced continuum damage mechanics models and has proved to reduce the computational effort by at least an order of magnitude as the modified linear momentum and phase-field equations are effectively linearised. As a result, the hybrid damage model is no more variationally consistent, but such a variational crime doesn't violate the second law of thermodynamics and the energy dissipation inequality as established in [89, 90], see also $[44,88,81]$. Moreover, the asymmetric nature of fracture under tensile/compressive deformation modes can be very accurately captured using the hybrid split while significantly enhancing the computational efficiency $[74,81,90]$.

\subsection{Implementation within Abaqus UMAT subroutine}

The solution algorithm is implemented within the commercial software Abaqus [91] using user-material (UMAT) subroutine as illustrated in Fig. 7. The choice of UMAT is based on its inherent advantage that most of the Abaqus in-built functionality, e.g., defining composite ply layups and fibre orientations, can be directly utilized. Moreover, no additional visualization modules are needed and the post-processed final results can be directly visualized in Abaqus CAE/Viewer as opposed to the user-element (UEL) subroutine, see e.g., [53, 54, 55, 56].

Since no additional D.O.Fs are introduced when using the UMAT subroutine, the Abaqus solver can only be used to solve those D.O.Fs which are inherent to the Abaqus standard library [91], e.g., the displacement D.O.Fs in this case. To solve the phase-field equations, an external linear system solver such as
Intel's Math-Kernel Library (MKL) Pardiso [92] is used in the current work. This is linked as an external library to the Abaqus platform at compilation. To facilitate the sequential solution of the linear momentum and phase-field equations within the staggered solution scheme, the UEXTERNALDB subroutine available in Abaqus is used to call both Abaqus and Pardiso solvers alternately in subsequent time-steps. The common variables which are required to solve both equations, e.g., the crack driving force $\tilde{\mathcal{D}}$ and the phase-field variable $\phi$, are exchanged via an external Fortran module. The damage results obtained at the end of each time-step are stored in the form of state-dependent variables STATEV to be used in the subsequent iterations.

\section{Experimental calibration}

The proposed anisotropic cohesive phase field model relies on the definition of the degradation functions defined in Eqs. (30)-(32). Hence, a proper calibration of all pertinent material parameters based on experimental measurements is required for the model to provide high-fidelity estimates. In particular, to fully define the degradation functions, the following parameters have to be identified (see also Fig. 8)

1. The critical fracture stresses $\sigma_{c i}$ corresponding to each damage mechanism $i \in\{11,22,12\}$ which defines the point of damage initiation;

2. The critical energy release rates $\mathcal{G}_{c i}$ which defines the total area under the cohesive traction-separation curve;

3. The shape of the cohesive softening curve, which is controlled by the parameters $\left\{m_{i}, p_{i}, q_{i}\right\}$ and;

4. The displacement at crack separation $\delta_{c i}$.

In practice, the model parameters $\left\{p_{i}, q_{i}\right\}$ for each damage mechanism $i \in\{11,22,12\}$ can be calibrated using two independent experiments. For the case of woven-fabric laminates, the value of the tensile parameters $\left\{p_{11}, q_{11}, p_{22}, q_{22}\right\}$ can be obtained from either an open-hole tension or a compact tension test of $\left(0^{\circ} / 90^{\circ}\right)$ laminates. The shear parameters $\left\{p_{12}, q_{12}\right\}$ can be obtained from a tensile test on $\left(-45^{\circ} / 45^{\circ}\right)$ laminates or a pure shear test on $\left(0^{\circ} / 90^{\circ}\right)$ laminates of the same material, see $[2,4]$ for more details.

Using the parametric definition of the traction separation law provided in Lorentz [71], within the context of a gradient enhanced continuum damage model, we define the following relations for each of the composite damage mechanism $i \in\{11,22,12\}$, i.e.,

$$
\frac{\sigma_{i}(\phi)}{\sigma_{c i}}=\tilde{\sigma}_{i}(\phi)
$$

where

$$
\tilde{\sigma}_{i}(\phi)=\frac{1-\phi}{\sqrt{A_{i}(\phi)}}
$$

and

$$
\frac{\sigma_{c i}}{\mathcal{G}_{c i}}\left[\delta_{i}(\phi)-L_{o} \frac{2 \sigma_{c i}}{E_{i}} \tilde{\sigma}_{i}(\phi)\right]=\tilde{\delta}_{i}(\phi),
$$




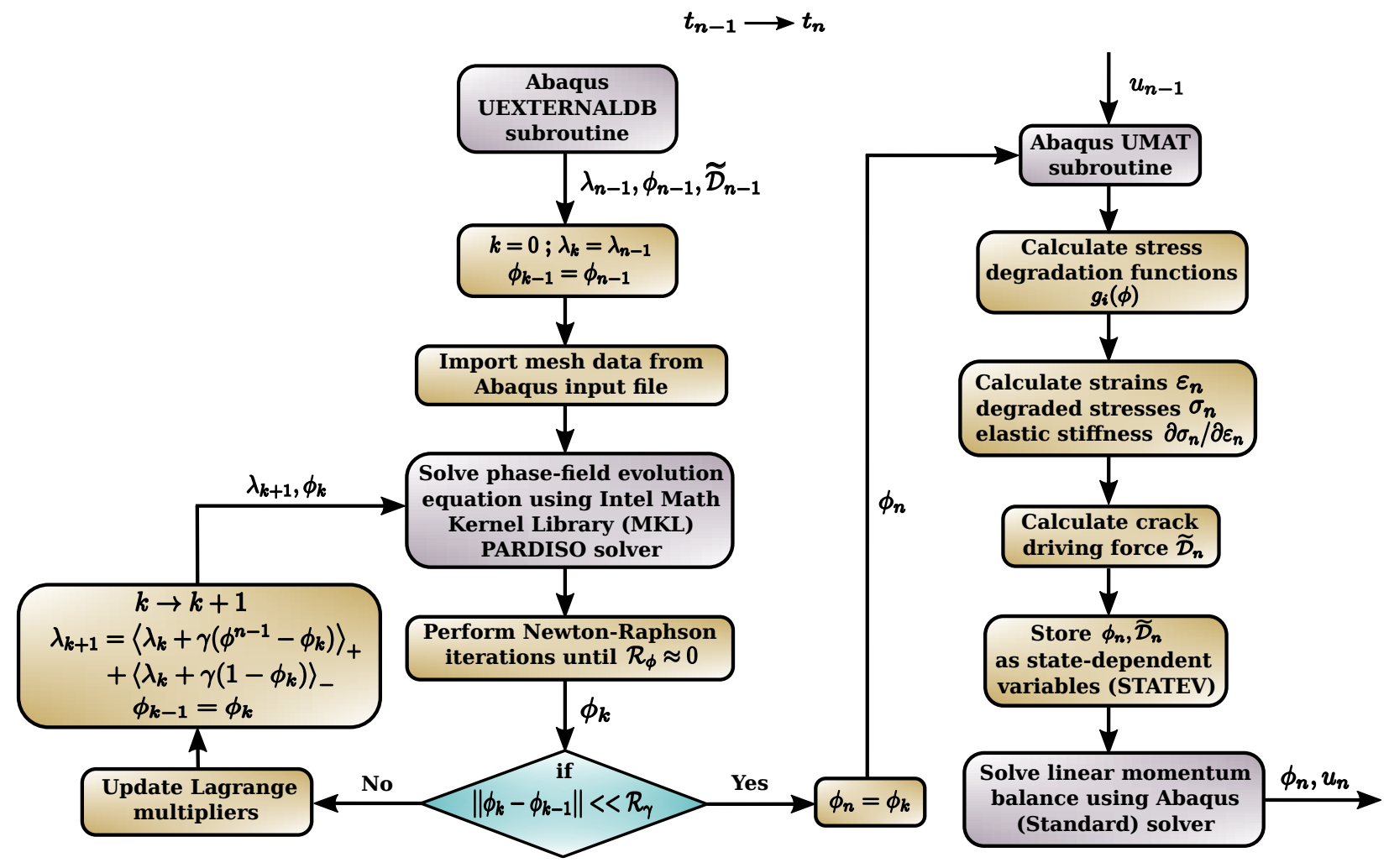

Figure 7: Flowchart illustrating solution procedure and algorithm implementation within Abaqus subroutines for time-increment $t_{n-1} \rightarrow t_{n}$

where

$$
\tilde{\delta}_{i}(\phi)=\frac{3}{2} \tilde{\sigma}_{i}(\phi) \int_{0}^{\phi} \frac{\sqrt{\phi^{\prime}} A_{i}\left(\phi^{\prime}\right)}{\left(1-\phi^{\prime}\right)^{2}}\left[1-\frac{A_{i}\left(\phi^{\prime}\right)(1-\phi)^{2}}{A_{i}(\phi)\left(1-\phi^{\prime}\right)^{2}}\right]^{-1 / 2} d \phi^{\prime}
$$

The analytical expressions Eqs. (59)-(62) can be fitted to experimentally derived softening curves hence identifying the anisotropic mode-specific material parameters for the cohesive phase field model.

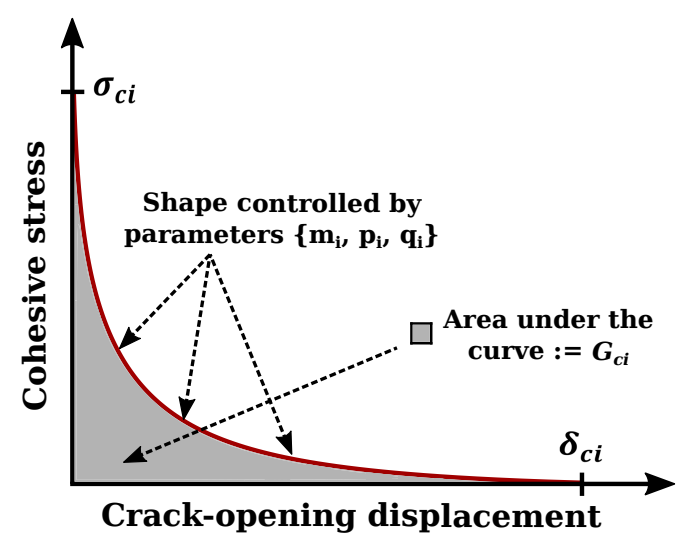

Figure 8: Illustration of cohesive softening law with associated material and fracture parameters for any specific composite damage mechanism $i$

\section{Applications}

6.1. Square plate with varying fibre orientation subjected to pure-tension

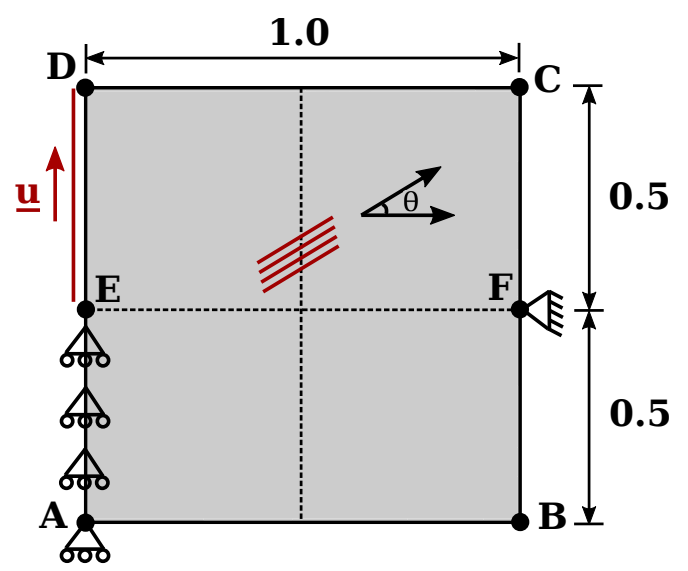

Figure 9: Anisotropic square plate under tensile loads: Geometry and boundary condition of square plate with an arbitrary fibre orientation $\theta$ [61]

A square plate under tensile loading is considered in this example. The geometry and boundary conditions are shown in Fig. 9. Node F is constrained in all translational and rotational DOFs. Only the vertical displacements off all the nodes on the edge AE are constrained. A uniform vertical displacement is imposed along the edge ED. This set of boundary conditions results in stress-concentration at $\mathrm{E}$, where the crack is expected 


\begin{tabular}{llll}
\hline Type & Property & Value & Unit \\
\hline Elastic properties & $E_{11}$ & 171000 & $\mathrm{MPa}$ \\
& $E_{22}$ & 9080 & $\mathrm{MPa}$ \\
& $G_{12}$ & 5290 & $\mathrm{MPa}$ \\
& $v_{12}$ & 0.32 & - \\
Fracture strength & $\sigma_{c 11}$ & 2325 & $\mathrm{MPa}$ \\
& $\sigma_{c 22}$ & 62.3 & $\mathrm{MPa}$ \\
Critical energy re- & $\mathcal{G}_{c 11}$ & 89.6 & $\mathrm{MPa}$ \\
lease rate & & 97.8 & $\mathrm{~N} / \mathrm{mm}$ \\
& $\mathcal{G}_{c 22}$ & 0.277 & $\mathrm{~N} / \mathrm{mm}$ \\
Cohesive softening & $\left(p_{11}, q_{11}\right)$ & $(1,0)$ & - \\
parameters & & & \\
& $\left(p_{22}, q_{22}\right)$ & $(1,0)$ & - \\
& $\left(p_{12}, q_{12}\right)$ & $(1,0)$ & - \\
\hline
\end{tabular}

Table 1: Elastic and fracture properties of IM7/8552 unidirectional ply taken from [93]

to initiate. The material properties considered are shown in Table 1 and correspond to a IM7/8552 unidirectional ply [14].

To examine the effect of the fibre orientation on the crack path, a set of simulations is performed for varying fibre angles, i.e., $\theta=0^{\circ}, 30^{\circ}, 45^{\circ}, 60^{\circ}$. To avoid the effect of meshbias, a uniform mesh is considered with 15750 elements and an element size $h_{e}=0.008 \mathrm{~mm}$. The length-scale parameter is $L=0.025 \mathrm{~mm}$. As shown in Fig. 10, the crack initiates at $\mathrm{E}$ and consistently propagates along the fibre directions for all orientations. This is consistent with experimental observations that in unidirectional composites, matrix cracking often becomes the primary mode of failure resulting in crack propagation parallel to fibres [77]. This is due to the fact that the matrix-strength is considerably lower than the fibre-strength, and the fracture energy required to crack the fibres (characterized by $\sigma_{c 11}$ and $\left.\mathcal{G}_{c 11}\right)$ is much higher than the fracture energy required to crack the matrix (characterized by $\sigma_{c 22}$ and $\mathcal{G}_{c 22}$ ).

\subsection{Compact-tension (CT) test for Textreme- $80 \mathrm{~g} / \mathrm{m}^{2}$ spread- tow fabric composite}

Compact-tension tests are typically performed to characterize the fibre tensile fracture toughness. In this example, we analyze the tensile fracture strength of a widely used spread-tow woven fabric-reinforced composite material, i.e., Textreme ${ }^{\circledR} 80$ $\mathrm{g} / \mathrm{m}^{2}$, which is manufactured by Oxeon [95]. An extensive experimental campaign was conducted on Textreme ${ }^{\circledR}[94,96,97]$, and the results obtained from Compact-Tension tests have been previously reported in [4]. In this example, we use the experimentally derived fracture response to validate the proposed anisotropic cohesive phase-field model.

The experiments were conducted on laminates each with a $\left[0^{\circ} / 90^{\circ}\right]$ stacking sequence containing 56 plies. The average laminate thickness was $4.37 \mathrm{~mm}$. A thin sharp crack with a radius of $250 \mu \mathrm{m}$ and a length of $10 \mathrm{~mm}$ was machined using a diamond coated wire. The loading was applied using a hydraulic universal testing machine MTS-858 which is equipped with a $25 \mathrm{kN}$ load cell, see Fig. 12. During the experiments, very little buckling was observed with the crack being confined in the desired crack path. In addition, no delamination was observed in the C-scan inspections which indicates the validity of the tests [94]. The reader is further referred to [4] for more details about the experimental procedure.

The geometry and boundary conditions employed in the simulation are shown in Fig. 11. The material properties are taken from [4] and shown in Table 2. Since the shear critical energy release rate $\mathcal{G}_{c 12}$ has not been provided in [4], it is assumed herein to be 1.5 times the tensile $\mathcal{G}_{c 11}$ and $\mathcal{G}_{c 22}$ owing to negligible shear cracking observed in Textreme ${ }^{\circledR} 80 \mathrm{~g} / \mathrm{m}^{2}$.

Remark 6. It of interest to note that the in-plane shear behaviour of fabric-reinforced composites is predominantly governed by the matrix properties [2]. However, due to the very small ply thickness and the fabric structure within the Textreme $^{\circledR} 80 \mathrm{~g} / \mathrm{m}^{2}$ laminate, there was negligible matrix cracking under in-plane shear observed during the experiments [4]. A similar behaviour was also reported by Wagih et al. [98], where fiber-based failures were the primary modes of intralaminar damage in Textreme ${ }^{\circledR} 80 \mathrm{~g} / \mathrm{m}^{2}$. Hence, the influence of the shear fracture properties is practically negligible on the overall fracture response.

Since there was no interlaminar damage observed in the experiments, the phase-field simulations are performed using a single layer of fully-integrated $\mathrm{S} 4$ shell elements available within the Abaqus standard library. The mesh consists of total 20320 elements and is refined with $h_{e}=0.1 \mathrm{~mm}$ in the central region where the crack is expected to propagate. The lengthscale parameter is taken as $L=0.6 \mathrm{~mm}$. Based on the chosen values of $h_{e}$ and $L$, the critical energy release rates provided in Table 2 are scaled to their effective values $\mathcal{G}_{c 11}=\mathcal{G}_{c 22}=54.5$ $\mathrm{N} / \mathrm{mm}$ and $\mathcal{G}_{c 12}=81.75 \mathrm{~N} / \mathrm{mm}$ based on Eq. (47). The evolution of the crack path with increasing load increments is shown in Fig. 13. The crack-path obtained from the cohesive phasefield model is compared with the experimentally obtained crack and C-Scan inspections in Fig. 14; the two crack paths match.

The influence of the cohesive parameters $p_{i}$ and $q_{i}$ on the critical fracture load and the post-fracture softening response for a fixed length-scale $L$ is illustrated in Fig. 15. It is evident that by choosing appropriate values of $p_{i}$ and $q_{i}$, a very accurate prediction of the experimental fracture response can be obtained, which also coincides with the prediction made by Soto et al. [4] using a Continuum Damage Mechanics (CDM) model, see Fig. 16.

Remark 7. The third set of cohesive parameters $m_{i}$ cannot be controlled independently for each individual composite damage mechanisms $i \in\{11,22,12\}$ as it inherently depends on the anisotropic material and fracture properties. Although different combinations of $m_{i} \in\left\{m_{11}, m_{22}, m_{12}\right\}$ can be obtained by varying the length-scale parameter $L$, each of these combinations would lead to strong interference of the anisotropic stressdegradation mechanisms. To qualitatively assess the influence of $L$ and $m_{i}$ on the overall fracture response, we consider an 


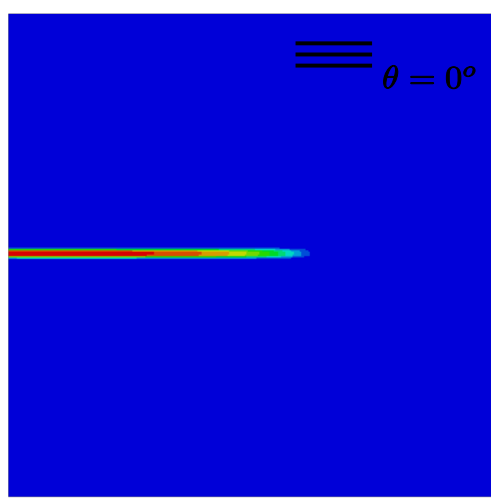

(a)
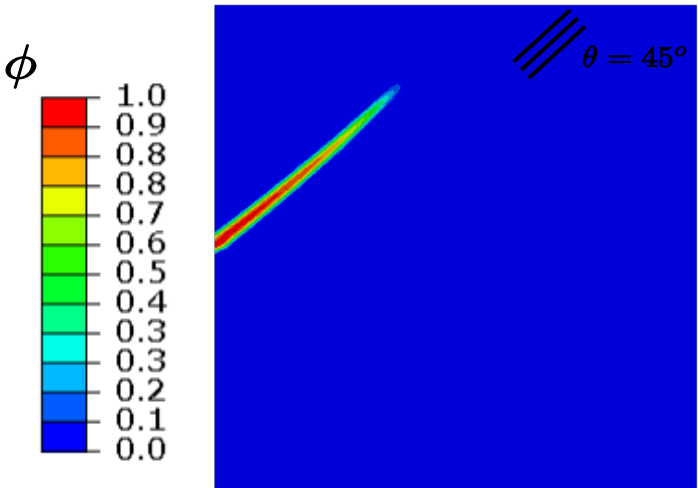

(c)

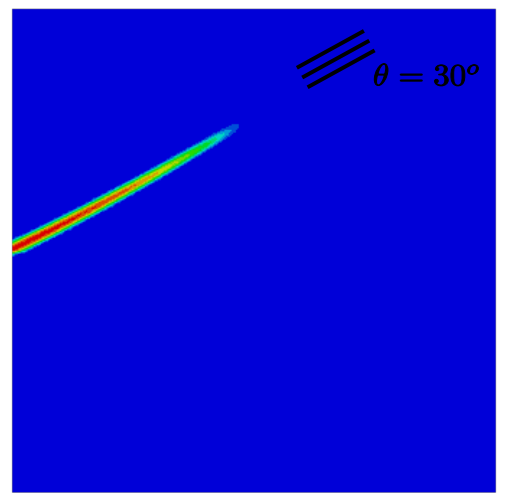

(b)

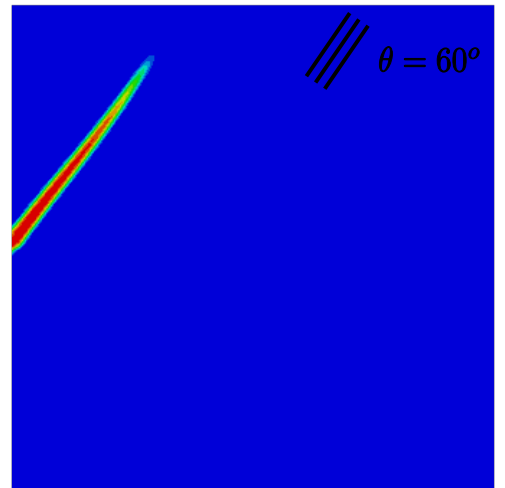

(d)

Figure 10: Anisotropic square plate under tensile loads: Crack propagation pattern with increasing time-increments for different fibre orientation angles $\theta=$ $0^{\circ}, 30^{\circ}, 45^{\circ}, 60^{\circ}$. The values of phase-field variable $\phi$ where $\phi=0$ and $\phi=1$ represent intact and cracked states of the material

\begin{tabular}{ll}
\hline Property & Values \\
\hline Elastic properties & $E_{11}=61400 \mathrm{MPa} ; E_{22}=61400 \mathrm{MPa} ; G_{12}=3782 \mathrm{MPa} ; v_{12}=0.042$ \\
Fracture strengths & $\sigma_{c 11}=975.4 \mathrm{MPa} ; \sigma_{c 22}=975.4 \mathrm{MPa} ; \sigma_{c 12}=85.9 \mathrm{MPa}$ \\
Shear plasticity parameters & $S_{L P}=30 \mathrm{MPa} ; \zeta_{E}=34.5 \mathrm{MPa} ; \zeta_{E}^{T}=141.5 ; \zeta_{L}=335.1 \mathrm{MPa}$ \\
Critical energy release rates & $\mathcal{G}_{c 11}=65.4 \mathrm{~N} / \mathrm{mm} ; \mathcal{G}_{c 22}=65.4 \mathrm{~N} / \mathrm{mm} ; \mathcal{G}_{c 12}=98.1 \mathrm{~N} / \mathrm{mm}$ \\
Cohesive phase-field parameters & $p_{11}=5.5 ; q_{11}=1.1 ; p_{22}=5.5 ; q_{22}=1.1 ; p_{12}=5.5 ; q_{12}=1.1 ; L=0.6 \mathrm{~mm}$ \\
\hline
\end{tabular}

Table 2: Elastic and fracture properties of Textreme ${ }^{\circledR} 80 \mathrm{~g} / \mathrm{m}^{2}$ taken from [4]

ideal isotropic case with $m_{i}=m_{11}=m_{22}=m_{12}=3 \mathcal{G}_{c} E / 2 L \sigma_{c}^{2}$. The CT simulations are then performed with different values of $m_{i}$ by modifying $L$, but fixing the values of $p_{i}=1$ and $q_{i}=0$.

The material and fracture properties for the isotropic case are taken as $E=61400 \mathrm{MPa}, \mathcal{G}_{c}=\mathcal{G}_{c 11}=\mathcal{G}_{c 22}=\mathcal{G}_{c 12}=54.5$ $\mathrm{N} / \mathrm{mm}$, and $\sigma_{c}=\sigma_{c 11}=\sigma_{c 22}=\sigma_{c 12}=975.4 \mathrm{MPa}$. All other input parameters are kept unchanged. The resulting crackpaths for different values of $\left[L, m_{i}\right]$ are shown in Fig. 18. The width of phase-field diffusion zone increases with increasing $L$ as expected. Furthermore, the influence of $\left[L, m_{i}\right]$ on the overall load-displacement response is illustrated in Fig. 17. It can be observed that the variations in either the length-scale $L$ or $m_{i}$ do not affect the resulting critical fracture loads and final crack separation, i.e., the initial and final stages of the softening curve; rather they only control its intermediate shape.

In the current analysis, $p_{i}=5.5, q_{i}=1.1$ and $L=0.6 \mathrm{~mm}$ have been found to provide a reasonably good approximation of the fracture response. In the next example, we will use these calibrated values of $\left\{p_{i}, q_{i}\right\}$ and $L$ to validate the experimental fracture response of Textreme ${ }^{\circledR} 80 \mathrm{~g} / \mathrm{m}^{2}$ using a double-edge notched tension (DENT) test.

\subsection{Double-edge notched tension (DENT) test for Textreme- $80 \mathrm{~g} / \mathrm{m}^{2}$ spread-tow fabric composite}

To assess the accuracy and predictive capabilities of the proposed phase-field model, we simulate a double-edge notched tension (DENT) experiment on a Textreme ${ }^{\circledR} 80 \mathrm{~g} / \mathrm{m}^{2}$ specimen using the calibrated material parameters of the previous example. We compare the simulation results with the experimental data [94].

DENT tests are typically performed on laminates to measure the crack resistance curve (R-curve) associated with their tensile fracture toughness. However for a cross-ply laminate like Textreme ${ }^{\circledR}$, DENT tests yield tensile fracture toughness corresponding to the fibers. The schematic of the DENT experiment 


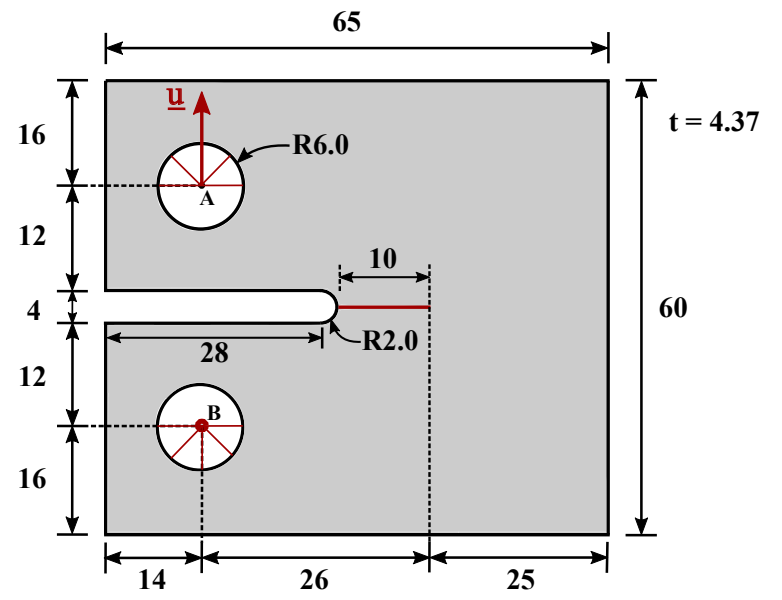

Figure 11: Compact Tension (CT) test: Geometry and boundary conditions for Textreme ${ }^{\circledR} 80 \mathrm{~g} / \mathrm{m}^{2}$ spread-tow fabric composite laminate [All dimensions in $\mathrm{mm}]$

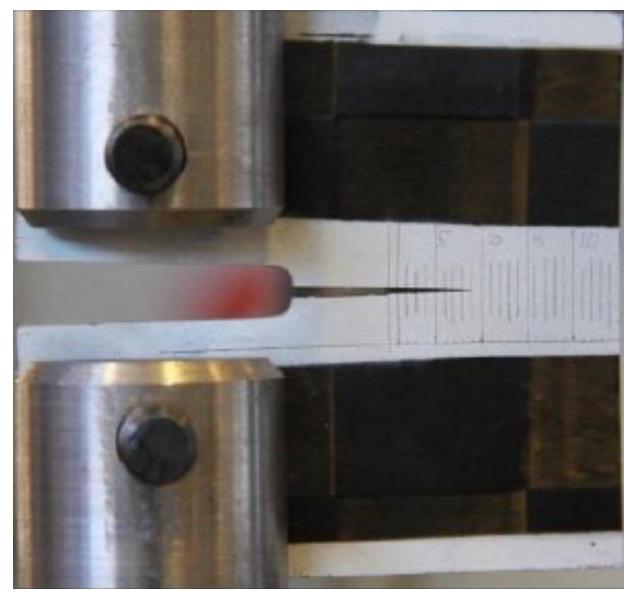

Figure 12: Compact Tension (CT) test: Experimental set-up and load application on Textreme ${ }^{\circledR} 80 \mathrm{~g} / \mathrm{m}^{2}$ spread-tow fabric composite laminate [94]

is illustrated in Fig. 19. The experiments were performed on a total 5 samples with different widths $(W)$ and initial notch lengths $\left(a_{0}\right)$, however the ratio $2 a_{0} / W$ was kept constant at a value $(\approx 0.6)$ for all samples [94]. The actual sizes of $W$ and $a_{0}$ used in the experiments are shown in Table 3, and the length of all samples is taken constant as $L=250 \mathrm{~mm}$. The laminate stacking sequence is $\left[0^{\circ} / 90^{\circ}\right]$ for all specimens with the total effective thicknesses as shown in Table 3. Similar to the CT specimens, a diamond coated wire was used to machine thin initial cracks on both left and right edges. The tests were performed with a hydraulic universal tester MTS-810 equipped with a $250 \mathrm{kN}$ load cell, and no tabs were used.

For the phase-field simulations, the material properties shown in Table 2 are used with the previously CT calibrated values of $\left\{p_{i}, q_{i}\right\}$ and $L$. The mesh is refined in the central region with $h_{e}=0.1 \mathrm{~mm}$. The total number of elements are 7044, 9422, 11815 and 14151 for specimens 1, 2, 3 and 4 respectively. The crack path and the representative load-displacement responses for the DENT specimens with varying widths and initial crack-lengths are shown in Fig. 20 and Fig. 21, respectively.
The predicted fracture strength are compared against the experimentally measured maximum loads in Table 3 . It is evident from Table 3 that the proposed phase-field model makes a very accurate prediction of the laminate fracture strength. It is worth noting that the values of $\left\{p_{i}, q_{i}, L\right\}$ can likewise be obtained for any other composite material, and are capable of accurately describing their quasi-brittle intralaminar fracture behaviour under different loading scenarios. Fig. 22 illustrates the size-effect law typically observed in woven fabric-reinforced composites. This occurs due to the presence of fracture process zone during quasi-brittle fractures, and the nominal and effective strengths of the laminate reduce with increasing specimen widths [99].

\subsection{Open-hole tension test on woven-fabric reinforced com- posite laminate}

An open-hole specimen with the geometry and boundary conditions shown in Fig. 23 is considered. We model a plain woven glass-reinforced fabric $\left[0^{\circ} / 90^{\circ}\right]$ embedded in an epoxy matrix with a fibre volume of $62 \%$, which was experimentally tested by Kim et al. [100] for a set of open-hole specimens. Martín-Santos et al. [2] compared the nominal strength predictions for this fabric composite with respect to experimental results using linear and bilinear Continuum Damage Mechanics (CDM) models, whereas Ahmad [101] used an XFEM model to analyze the nominal strength. It was observed in [2] that the fabric exhibits a quasi-brittle fracture response with a large decrease in stresses but minimal crack-opening post-initiation, followed by a zone defined by a smaller decrease in stresses but large crack opening displacements. Hence, a bilinear CDM model was found to be most suitable for capturing the quasibrittle behaviour and predicting the nominal strength of the fabric accurately. In the current work, we perform the open-hole tension test on specimens of varying width $W$ and hole-diameter $D$ using the proposed cohesive phase-field model and compare the nominal strength predictions with results from experiments in [100] and bilinear CDM model in [2].

The material properties are taken from $[100,2]$ and are also shown in Table 4 for reference. The ply lay-up is $\left[0^{\circ} / 90^{\circ}\right]$, and the mesh is refined in the central zone with $h_{e}=0.2 \mathrm{~mm}$. The length-scale parameter is chosen as $L=1.2 \mathrm{~mm}$. The mesh for specimens 1, 2, 3 and 4 comprise 3480, 2621, 6780 and 4527 S4 shell elements respectively. Based on these values, the critical energy release rates in [100] can be scaled to their effective values $\mathcal{G}_{c 11}=\mathcal{G}_{c 22}=47.92 \mathrm{~N} / \mathrm{mm}$ and $\mathcal{G}_{c 12}=71.875 \mathrm{~N} / \mathrm{mm}$ as described in Eq. (47). The specimen with $W=20 \mathrm{~mm}$ and $D=8 \mathrm{~mm}$ is used to calibrate the softening parameters $\left\{p_{i}, q_{i}\right\}$ required by the cohesive phase-field model as shown in Fig. 24 and Table 5. These are then used to predict and validate the experimentally obtained nominal strengths for remaining specimens.

It can be deduced from Fig. 24 and Table 5 that the parameters $p_{i}=1$ and $q_{i}=0$ provide the most accurate match with the experimental laminate fracture strength. The crack-paths for all tested samples are shown in Fig. 25, wherein two crackbranches initiate simultaneously at diametrically opposite ends of the hole and propagate horizontally towards the side-edges. This is consistent with experimental results in the literature 


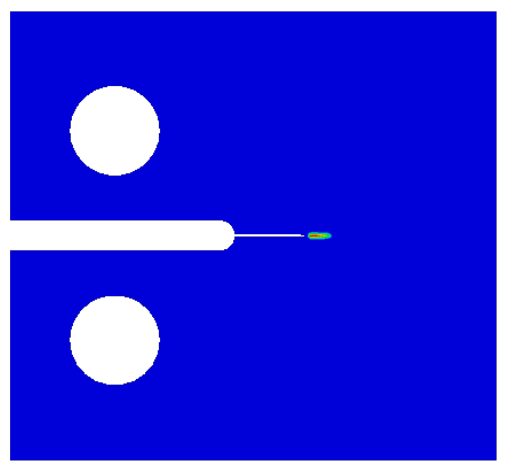

(a)

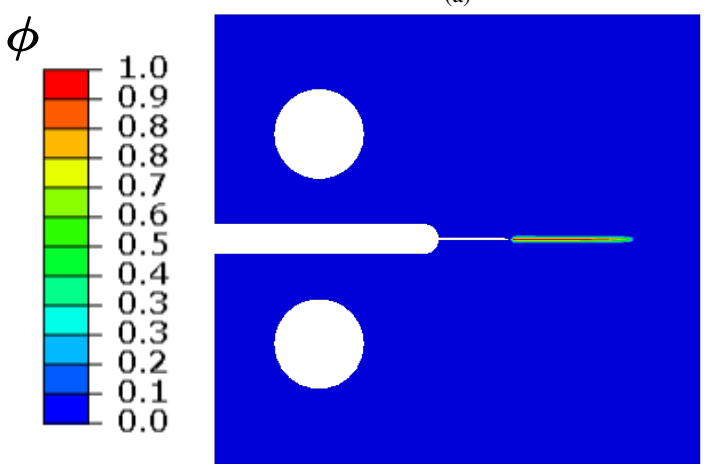

(c)

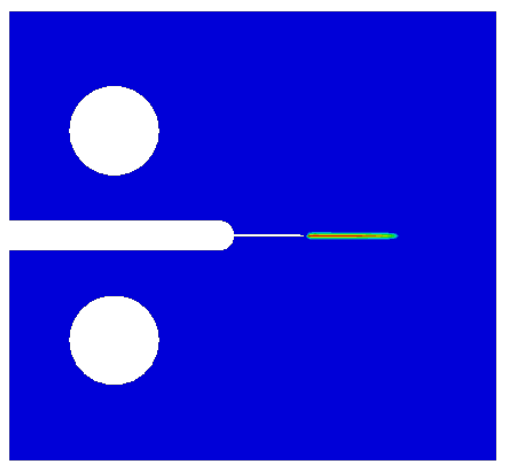

(b)

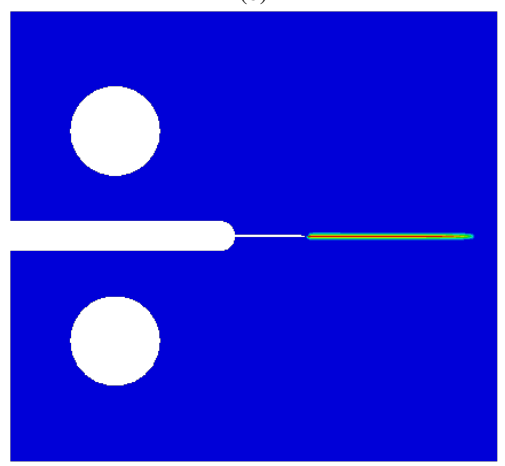

(d)

Figure 13: Compact-tension (CT) test on Textreme ${ }^{\circledR} 80 \mathrm{~g} / \mathrm{m}^{2}$ : Crack propagation pattern for increasing time-increments with cohesive phase-field parameters $p_{i}=5.5, q_{i}=1.1, L=0.6 \mathrm{~mm}$. The values of phase-field variable $\phi=0$ and $\phi=1$ represent intact and cracked states of the material

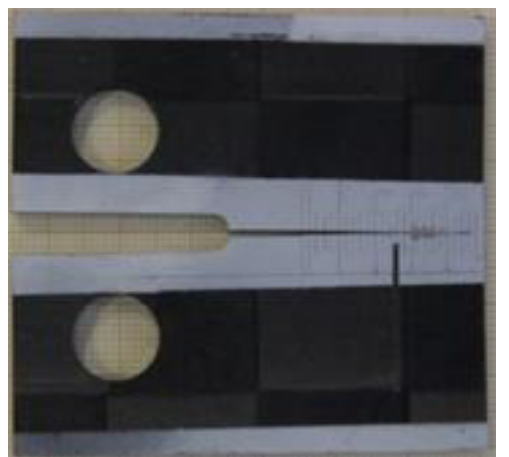

(a)

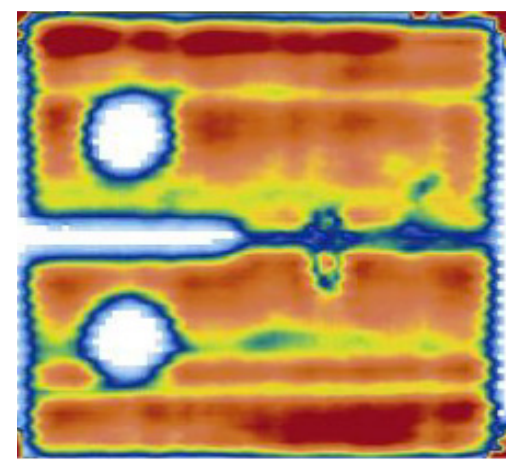

(b)

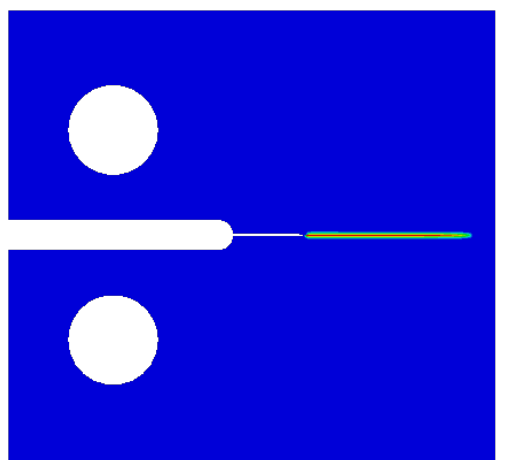

(c)

Figure 14: Compact-tension (CT) test on Textreme ${ }^{\circledR} 80 \mathrm{~g} / \mathrm{m}^{2}$ : Comparison of crack-paths obtained from (a) Experiments [94, 97] (b) C-Scan inspection [97] (c) Cohesive phase-field model

$[102,63]$. The load-displacement response for specimens with different combinations of widths $W$ and hole diameters $D$ is shown in Fig. 26.

The nominal strengths predicted by the cohesive phase-field model are compared with the experimental data [100] and CDM bilinear model [2] in Table 6 . The calibrated cohesive phasefield model provides a very good prediction of the nominal strengths for all specimens with percentage errors lying within $3-4 \%$, which highlights the robust predictive capability of the proposed model.

\subsubsection{Mesh convergence study}

To investigate the sensitivity of the resulting critical fracture loads and the corresponding crack patterns on the meshsize $h_{e}$ and the length-scale parameter $L$, we perform a meshconvergence study on the specimen with dimensions $W=$ $20 \mathrm{~mm}$ and $D=4 \mathrm{~mm}$. Three different mesh-sizes are considered, i.e., $h_{e}=0.1 \mathrm{~mm}, h_{e}=0.15 \mathrm{~mm}$ and $h_{e}=0.2 \mathrm{~mm}$. The length-scales are chosen as $L=0.6 \mathrm{~mm}, L=0.9 \mathrm{~mm}$ and $L=1.2 \mathrm{~mm}$ respectively, thus retaining a constant ratio $h_{e} / L=1 / 6$ to ensure that the effective critical energy release rates $\mathcal{G}_{c i}$ in Eq. (47) are scaled by the same amount.

The resulting load-displacement plots are shown in Fig. 28 and demonstrate that the critical fracture force and the overall 


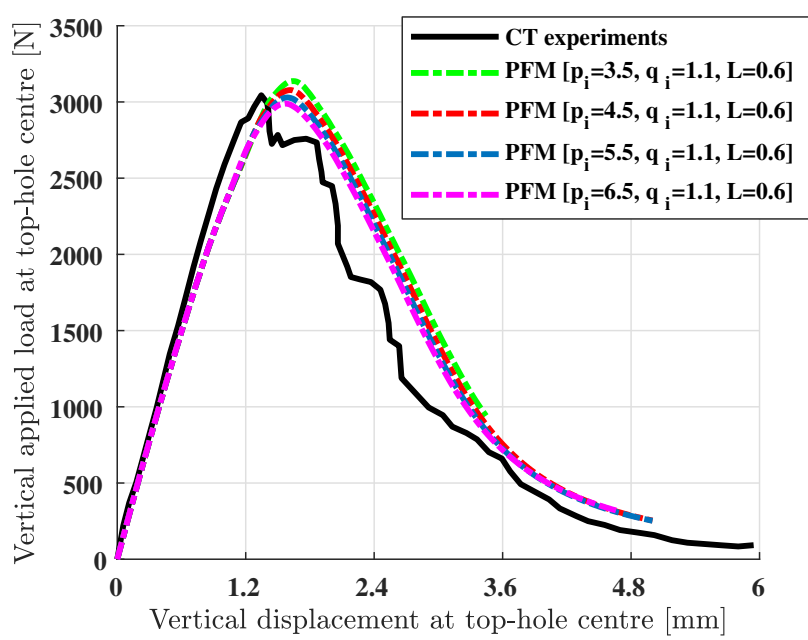

(a)

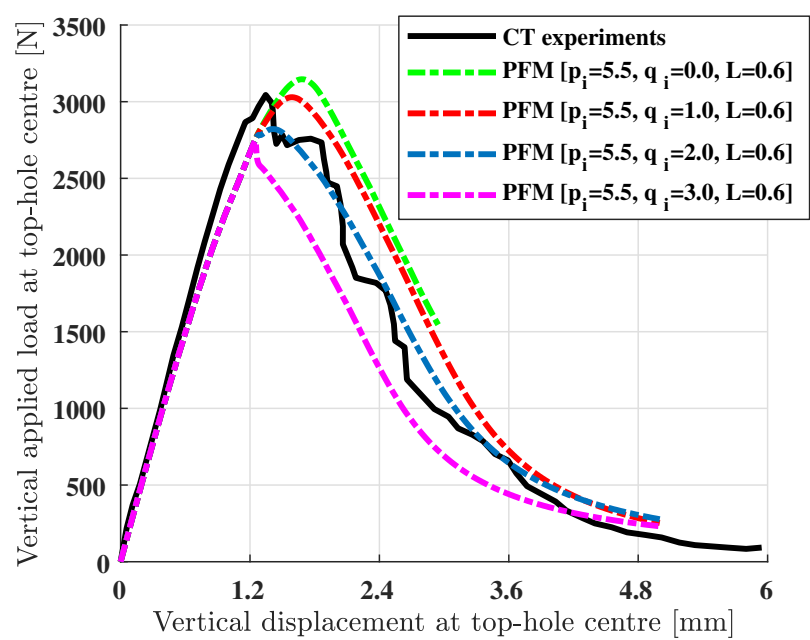

(b)

Figure 15: Compact-tension (CT) test on Textreme ${ }^{\circledR} 80 \mathrm{~g} / \mathrm{m}^{2}$ : Effect of parameters (a) $p_{i}$, and (b) $q_{i}$ on the overall load-displacement response

\begin{tabular}{|c|c|c|c|c|c|c|c|c|c|}
\hline $\begin{array}{l}\text { Specimen } \\
\text { number }\end{array}$ & $\begin{array}{l}\text { Mean } \\
\text { nominal } \\
\text { width W } \\
{[\mathrm{mm}]}\end{array}$ & $\begin{array}{l}\text { Mean } \\
\text { crack- } \\
\text { length } \\
2 a_{0}[\mathrm{~mm}]\end{array}$ & $\begin{array}{l}\text { Mean } \\
\text { effective } \\
\text { width } \\
{[\mathrm{mm}]}\end{array}$ & $\begin{array}{l}\text { Laminate } \\
\text { thickness } \\
t[\mathrm{~mm}]\end{array}$ & 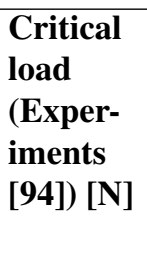 & $\begin{array}{l}\text { Critical } \\
\text { load (Co- } \\
\text { hesive } \\
\text { PFM) } \\
{[\mathrm{N}]}\end{array}$ & 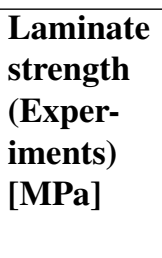 & $\begin{array}{l}\text { Laminate } \\
\text { strength } \\
\text { (Co- } \\
\text { hesive } \\
\text { PFM) } \\
\text { [MPa] }\end{array}$ & $\begin{array}{l}\text { Absolute } \\
\text { pre- } \\
\text { diction } \\
\text { error } \\
{[\%]}\end{array}$ \\
\hline 1 & 15 & 9.1 & 5.9 & 4.36 & 12522.9 & 12979 & 191.92 & 198.91 & $3.6 \%$ \\
\hline 2 & 20.1 & 12.2 & 7.9 & 4.37 & 15181.7 & 15471.3 & 172.84 & 176.14 & $1.9 \%$ \\
\hline 3 & 24.9 & 15.1 & 9.8 & 4.38 & 17763 & 17955.7 & 162.87 & 164.64 & $1.08 \%$ \\
\hline 4 & 30.1 & 18.2 & 11.9 & 4.41 & 20288.5 & 20277.2 & 152.84 & 152.76 & $0.05 \%$ \\
\hline
\end{tabular}

Table 3: Double-edge notched tension (DENT) test on Textreme ${ }^{\circledR} 80 \mathrm{~g} / \mathrm{m}^{2}$ : Summary of specimen sizes used for the experiments [94], and comparison between critical fracture loads and strengths obtained from the experiments and the proposed cohesive phase-field model $(\mathrm{PFM})$ with parameters $p_{i}=5.5, q_{i}=1.1$ and length-scale $L=0.6 \mathrm{~mm}$

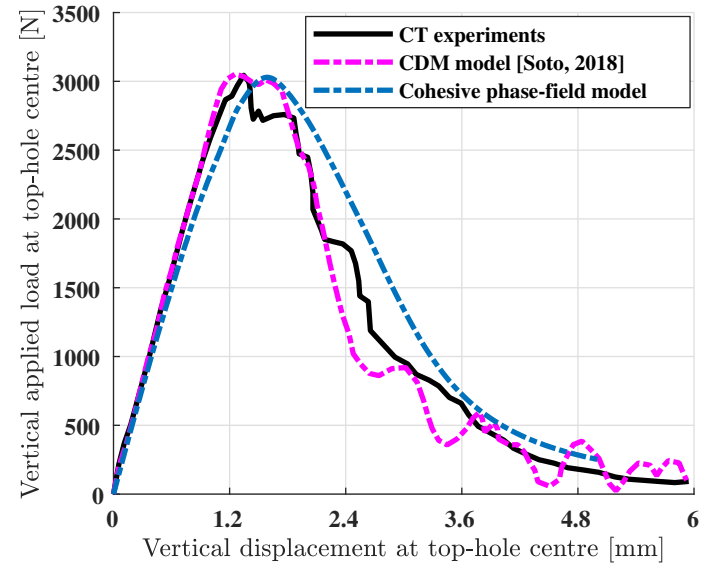

Figure 16: Compact-tension (CT) test on Textreme ${ }^{\circledR} 80 \mathrm{~g} / \mathrm{m}^{2}$ : Loaddisplacement response obtained from the cohesive phase-field model with parameters $\left[p_{i}=5.5, q_{i}=1.1, L=0.6\right]$. Also shown is its comparison with $\mathrm{CT}$ experimental response and the prediction made using continuum damage mechanics (CDM) model in [4]

load-displacement response remain identical in all cases. The corresponding crack-paths are shown in Fig. 27. As the length

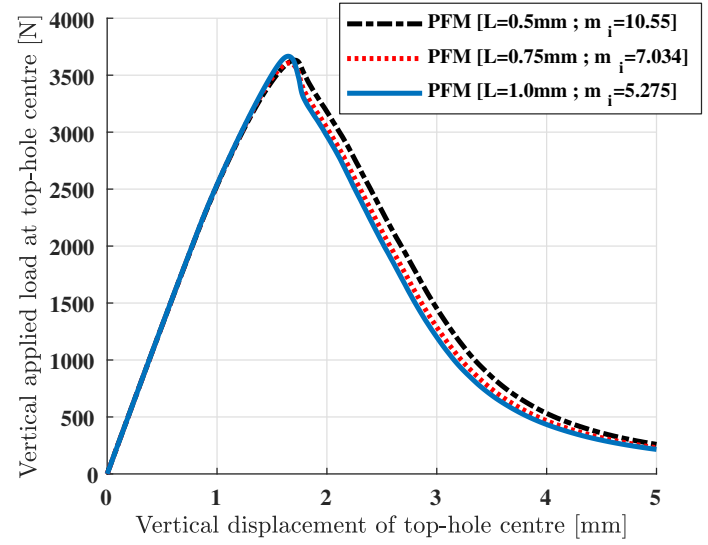

Figure 17: Compact-tension (CT) test on Textreme ${ }^{\circledR} 80 \mathrm{~g} / \mathrm{m}^{2}$ : Effect of parameters $\left[L, m_{i}\right]$ on the overall load-displacement response with (a) $L=0.5 \mathrm{~mm}$ ; $m_{i}=10.55$ (black) (b) $L=0.75 \mathrm{~mm} ; m_{i}=7.034$ (red) (c) $L=1.0 \mathrm{~mm}$; $m_{i}=5.275$ (blue). The comparison is made assuming an ideal isotropic case with $m_{i}=m_{11}=m_{22}=m_{12}$ and $\left[p_{i}=1 ; q_{i}=0\right]$

scale increases, the diffusion width of the crack increases although without affecting the load bearing capacity of the simu- 


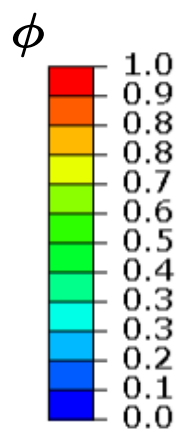

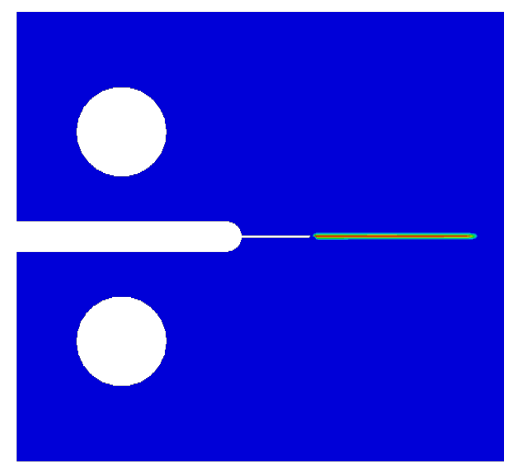

(a)

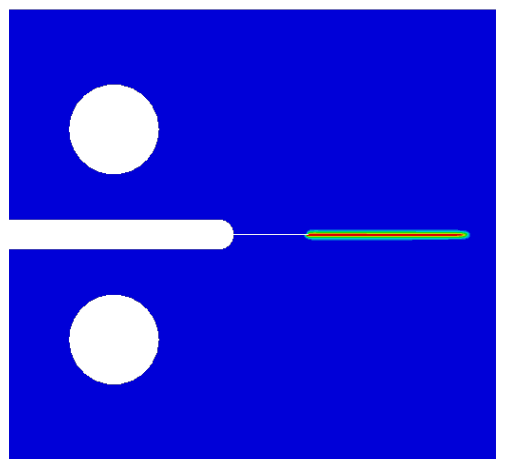

(b)

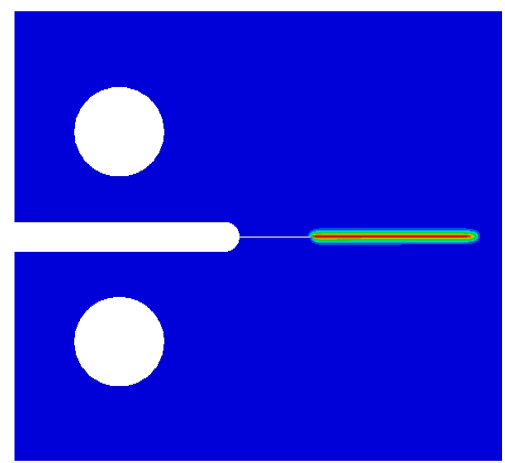

(c)

Figure 18: Compact-tension (CT) test on Textreme ${ }^{\circledR} 80 \mathrm{~g} / \mathrm{m}^{2}$ : Influence of length-scale parameter $\left[L, m_{i}\right]$ on the phase-field diffusion width for (a) $L=0.5 \mathrm{~mm}$; $m_{i}=10.55$ (b) $L=0.75 \mathrm{~mm} ; m_{i}=7.034$ (c) $L=1.0 \mathrm{~mm} ; m_{i}=5.275$. The values of phase-field variable $\phi=0$ and $\phi=1$ represent intact and cracked states of the material. Also, the comparison is made assuming an ideal isotropic case with $m_{i}=m_{11}=m_{22}=m_{12}$ and $\left[p_{i}=1 ; q_{i}=0\right]$

\begin{tabular}{ll}
\hline Property & Values \\
\hline Elastic properties & $E_{11}=23600 \mathrm{MPa} ; E_{22}=23600 \mathrm{MPa} ; G_{12}=4000 \mathrm{MPa} ; v_{12}=0.11$ \\
Fracture strengths & $\sigma_{c 11}=351.4 \mathrm{MPa} ; \sigma_{c 22}=351.4 \mathrm{MPa} ; \sigma_{c 12}=351.4 \mathrm{MPa}$ \\
Shear plasticity parameters & $S_{L P}=121 \mathrm{MPa} ; \zeta_{E}=5 \mathrm{MPa} ; \zeta_{E}^{T}=500 ; \zeta_{L}=10 \mathrm{MPa}$ \\
Critical energy release rates & $\mathcal{G}_{c 11}=57.5 \mathrm{~N} / \mathrm{mm} ; \mathcal{G}_{c 22}=57.5 \mathrm{~N} / \mathrm{mm} ; \mathcal{G}_{c 12}=86.25 \mathrm{~N} / \mathrm{mm}$ \\
Cohesive phase-field parameters & $p_{11}=1.0 ; q_{11}=0.0 ; p_{22}=1.0 ; q_{22}=0.0 ; p_{12}=1.0 ; q_{12}=0.0 ; L=1.2 \mathrm{~mm}$ \\
\hline
\end{tabular}

Table 4: Elastic and fracture properties of woven glass-reinforced fabric embedded in an epoxy matrix obtained from [100, 2] for Open-Hole Tension $($ OHT) test

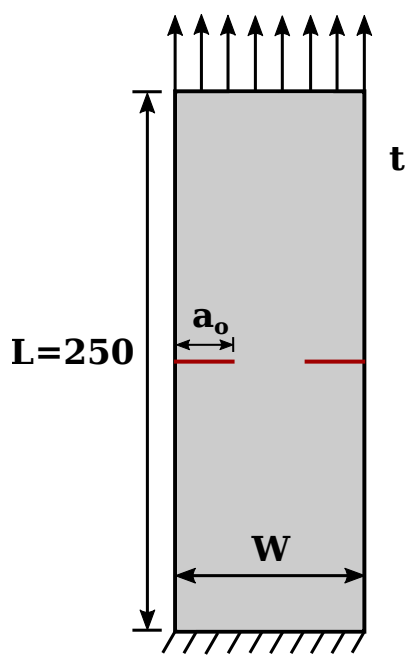

Figure 19: Double-edge notched tension (DENT) test: Geometry and boundary conditions for Textreme ${ }^{\circledR} 80 \mathrm{~g} / \mathrm{m}^{2}$ spread-tow fabric composite laminate [All dimensions in $\mathrm{mm}]$

lated specimen.

\subsection{Open-hole tension test on unidirectional (UD) composite laminate}

The case of a unidirectional (UD) composite material is examined herein. An open-hole tension test on a quasi-UD flax fiber/epoxy matrix composite FlaxPly-UD180 is considered. The geometry and loading conditions are shown in Fig. 29. The areal density of the composite is $180 \mathrm{~g} / \mathrm{m}^{2}$ [105]. The material properties are taken from $[103,104]$ and are provided in Table 7

\begin{tabular}{llllll}
\hline$p_{i}$ & $q_{i}$ & $L$ & $\begin{array}{l}\text { Laminate } \\
\text { strength } \\
\text { (Exper- } \\
\text { iments) } \\
{[\mathbf{M P a}]}\end{array}$ & $\begin{array}{l}\text { Predicted } \\
\text { numerical } \\
\text { strength } \\
{[\mathbf{M P a}]}\end{array}$ & $\begin{array}{l}\text { Absolute } \\
\text { predic- } \\
\text { tion error } \\
{[\%]}\end{array}$ \\
\hline 1 & 0 & 1.2 & 138 & 139.67 & $1.21 \%$ \\
\hline 2 & 0 & 1.2 & 138 & 131.9 & $4.42 \%$ \\
\hline 3 & 0 & 1.2 & 138 & 126 & $8.7 \%$ \\
\hline
\end{tabular}

Table 5: Open-hole tension (OHT) test on woven glass-reinforced fabric composite: Laminate strengths predicted using different combinations of cohesive phase-field parameters for specimen with width $W=20 \mathrm{~mm}$ and holediameter $D=8 \mathrm{~mm}$, and their comparison with experimental laminate strength $[100,2]$.The most accurate numerical response is obtained using parameters $\left[p_{i}=1, q_{i}=0, L=1.2 \mathrm{~mm}\right]$

for reference. A refined mesh is employed with $h_{e}=0.2 \mathrm{~mm}$, and the length-scale parameter is assumed $L=1.2 \mathrm{~mm}$.

The critical energy release rates $\left(\mathcal{G}_{c 22}\right.$ and $\left.\mathcal{G}_{c 12}\right)$ for transverse tensile and shear matrix cracking are provided in [103, 104], however $\mathcal{G}_{c 11}$ responsible for tensile fibre-failure is not provided. Hence, $\mathcal{G}_{c 11}$ is assumed to be 50 times the magnitude of $\mathcal{G}_{c 22}$ to prevent fibre-failure in accordance with the assumption in [76]. Similarly, the transverse shear $\sigma_{c 12}$ and longitudinal tensile strengths $\sigma_{c 11}$ are assumed based on the transverse tensile strength $\sigma_{c 22}=20.25 \mathrm{MPa}$ provided in [104] using the expressions below:

$$
\sigma_{c 11}=\sqrt{\sigma_{c 22}^{2} \frac{\mathcal{G}_{c 11}}{\mathcal{G}_{c 22}}}=143.19 \mathrm{MPa}
$$




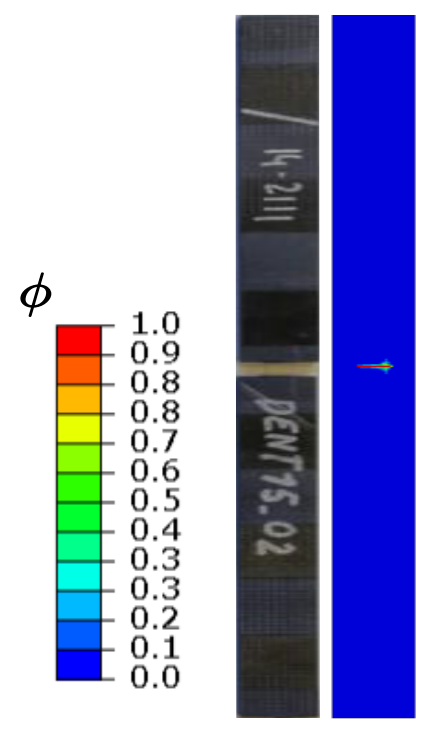

(a)

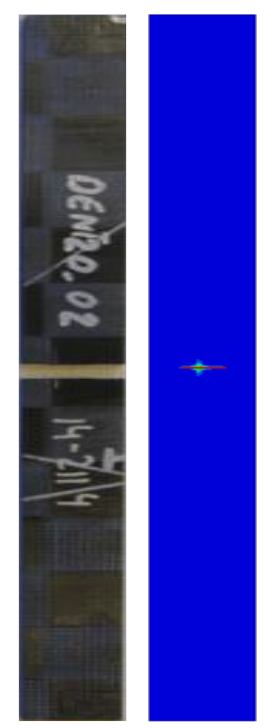

(b)

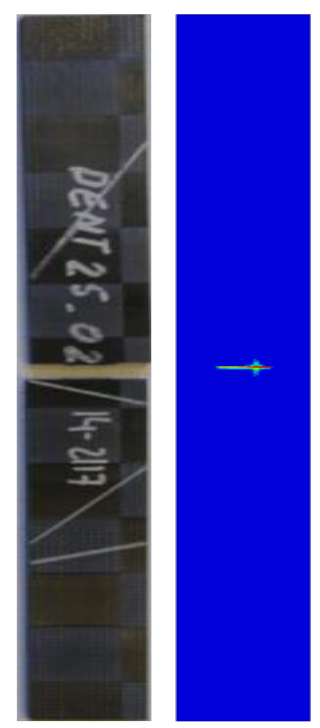

(c)
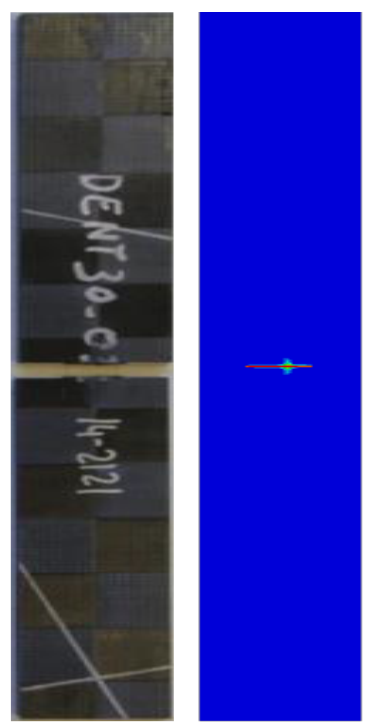

(d)

Figure 20: Double edge notched tension (DENT) test on Textreme ${ }^{\circledR} 80 \mathrm{~g} / \mathrm{m}^{2}$ : Crack paths obtained from experiments [94] and the cohesive phase-field model with parameters $p_{i}=5.5, q_{i}=1.1, L=0.6 \mathrm{~mm}$ for specimens with (a) Specimen- $\left[W=15 \mathrm{~mm} ; 2 a_{0}=9.1 \mathrm{~mm}\right]$ (b) Specimen-2 $\left[W=20.1 \mathrm{~mm} ; 2 a_{0}=12.2 \mathrm{~mm}\right](\mathrm{c})$ Specimen-3 $\left[W=24.9 \mathrm{~mm} ; 2 a_{0}=15.1 \mathrm{~mm}\right]$ (d) Specimen-4 $\left[W=30.1 \mathrm{~mm} ; 2 a_{0}=18.2 \mathrm{~mm}\right]$. The values of phase-field variable $\phi=0$ and $\phi=1$ represent intact and cracked states of the material

\begin{tabular}{llllllllll}
\hline $\begin{array}{l}\text { Specimen } \\
\text { number }\end{array}$ & $\begin{array}{l}\text { Mean } \\
\text { width } \\
{[\mathbf{m m}]}\end{array}$ & $\begin{array}{l}\text { Hole di- } \\
\text { ameter } \mathbf{D} \\
{[\mathbf{m m}]}\end{array}$ & $\begin{array}{l}\text { Laminate } \\
\text { thickness } \\
{[\mathbf{m m}]}\end{array}$ & $\begin{array}{l}\text { Laminate } \\
\text { strength (Ex- } \\
\text { periments } \\
{[\mathbf{1 0 0 ]}[\mathbf{M P a}]}\end{array}$ & $\begin{array}{l}\text { Laminate } \\
\text { strength } \\
\text { (CDM [2]) } \\
{[\mathbf{M P a}]}\end{array}$ & $\begin{array}{l}\text { Critical } \\
\text { load (Co- } \\
\text { hesive } \\
\text { PFM) [N] }\end{array}$ & $\begin{array}{l}\text { Laminate } \\
\text { strength } \\
\text { (Cohesive } \\
\text { PFM) [MPa] }\end{array}$ & $\begin{array}{l}\text { Absolute } \\
\text { predic- } \\
\text { tion error } \\
{[\%]}\end{array}$ \\
\hline 1 & 20 & 4.0 & 2.0 & 192.9 & 193.65 & 8034 & 200.85 & $4.12 \%$ \\
\hline 2 & 20 & 8.0 & 2.0 & 138 & 136.85 & 5587 & 139.675 & $1.21 \%$ \\
\hline 3 & 40 & 8.0 & 2.0 & 167.5 & 169.94 & 13450 & 168.125 & $0.37 \%$ \\
\hline 4 & 40 & 16.0 & 2.0 & 121.6 & 123.78 & 9391 & 117.3875 & $3.46 \%$ \\
\hline
\end{tabular}

Table 6: Open-hole tension (OHT) test on woven glass-reinforced fabric composite: Summary of experimental specimen sizes as obtained from [100, 2], and comparison between critical fracture strengths obtained from the experiments and the proposed cohesive phase-field model (PFM) with parameters $p_{i}=1.0, q_{i}=0$ and length-scale $L=1.2 \mathrm{~mm}$

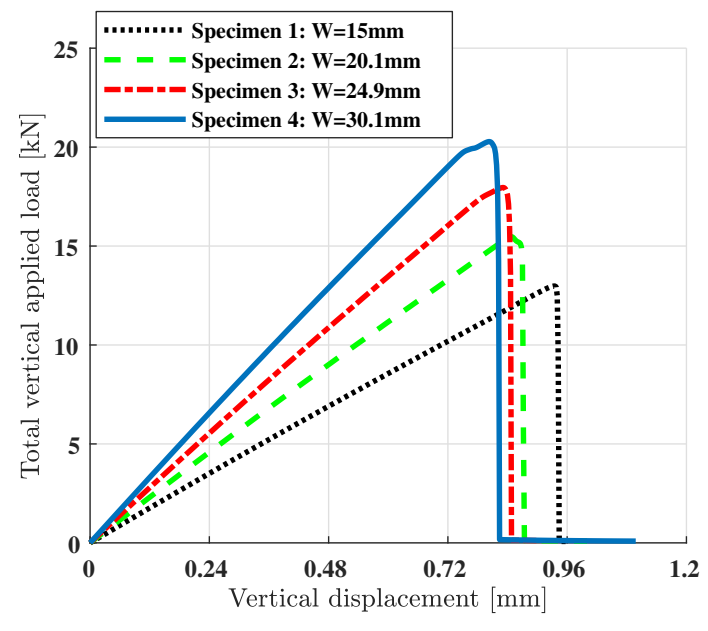

Figure 21: Double edge notched tension (DENT) test on Textreme ${ }^{\circledR} 80 \mathrm{~g} / \mathrm{m}^{2}$ : Load-displacement responses for varying specimen widths obtained from the cohesive phase-field model with parameters $\left[p_{i}=5.5, q_{i}=1.1, L=0.6\right]$

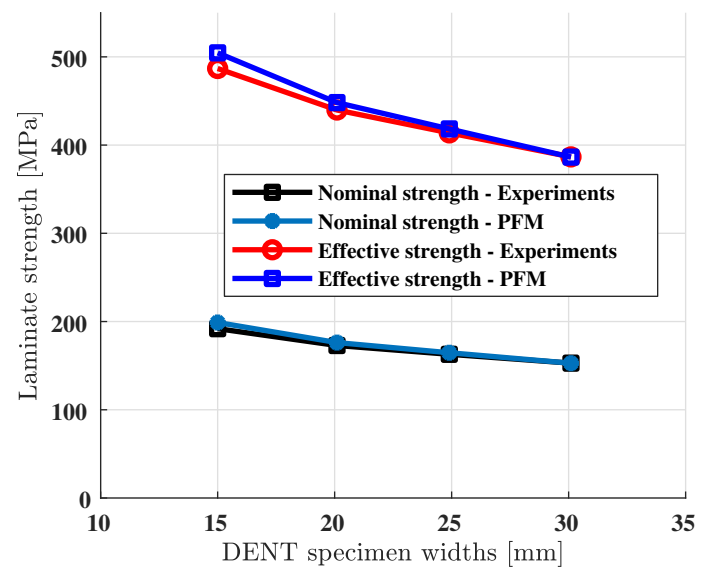

Figure 22: Double edge notched tension (DENT) test on Textreme ${ }^{\circledR} 80 \mathrm{~g} / \mathrm{m}^{2}$ : Strength vs specimen widths obtained from experiments [94] and the cohesive phase-field model illustrating the size-effect law in fabric-reinforced composites. Nominal and effective strengths have been calculated based on the total and effective laminate widths respectively 


\begin{tabular}{ll}
\hline Property & Values \\
\hline Elastic properties & $E_{11}=26500 \mathrm{MPa} ; E_{22}=2600 \mathrm{MPa} ; G_{12}=1300 \mathrm{MPa} ; v_{12}=0.35$ \\
Fracture strengths & $\sigma_{c 11}=143.19 \mathrm{MPa}$ (assumed); $\sigma_{c 22}=20.25 \mathrm{MPa} ; \sigma_{c 12}=17.64 \mathrm{MPa}$ (assumed) \\
Critical energy release rates & $\mathcal{G}_{c 11}=31.1 \mathrm{~N} / \mathrm{mm}$ (assumed) $; \mathcal{G}_{c 22}=0.622 \mathrm{~N} / \mathrm{mm} ; \mathcal{G}_{c 12}=0.472 \mathrm{~N} / \mathrm{mm}$ \\
Cohesive phase-field parameters & $p_{11}=1.0 ; q_{11}=0.0 ; p_{22}=1.0 ; q_{22}=0.0 ; p_{12}=1.0 ; q_{12}=0.0 ; L=1.2 \mathrm{~mm}$ \\
\hline
\end{tabular}

Table 7: Elastic and fracture properties for Open-Hole Tension (OHT) test on quasi-unidirectional (UD) flax fiber/epoxy matrix composite FlaxPly-UD180 obtained from $[103,104]$

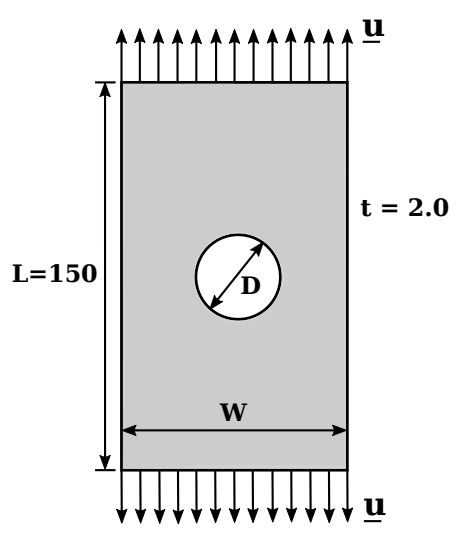

Figure 23: Open-hole tension (OHT) test: Geometry and boundary conditions for a plain woven glass-reinforced fabric composite [100, 2] [All dimensions in $\mathrm{mm}]$

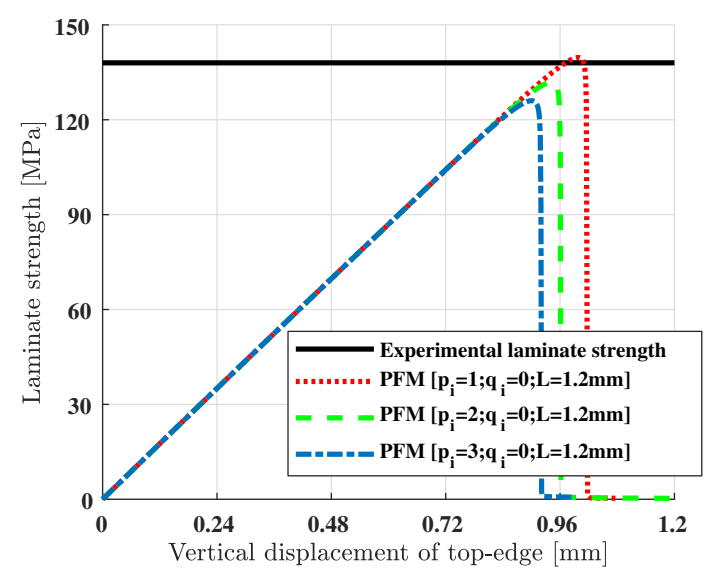

Figure 24: Open-hole tension (OHT) test on woven glass-reinforced fabric composite: Calibration of cohesive phase-field parameters with respect to the experimental laminate strength in $[100,2]$ for specimen with width $W=20 \mathrm{~mm}$ and hole-diameter $D=8 \mathrm{~mm}$. The most accurate numerical response is obtained using parameters $\left[p_{i}=1, q_{i}=0\right]$. The length scale is $L=1.2 \mathrm{~mm}$ in all cases.

$$
\sigma_{c 12}=\sqrt{\sigma_{c 22}^{2} \frac{\mathcal{G}_{c 12}}{\mathcal{G}_{c 22}}}=17.64 \mathrm{MPa}
$$

Considering $h_{e}=0.2 \mathrm{~mm}$ and $L=1.2 \mathrm{~mm}$, the critical energy release rates are scaled to their effective values based on Eq. (47) as $\mathcal{G}_{c 11}=25.9167 \mathrm{~N} / \mathrm{mm}, \mathcal{G}_{c 22}=0.5183 \mathrm{~N} / \mathrm{mm}$ and $\mathcal{G}_{c 12}=0.393 \mathrm{~N} / \mathrm{mm}$. Since unidirectional composites do not typically display plasticity in their shear behaviour, the shear plasticity parameters are omitted in this case.

A series of analyses is performed considering different fibre-

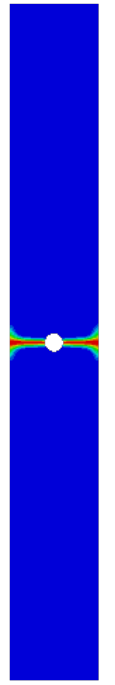

(a)

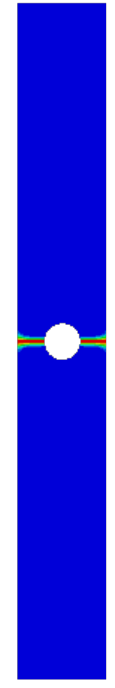

(b)

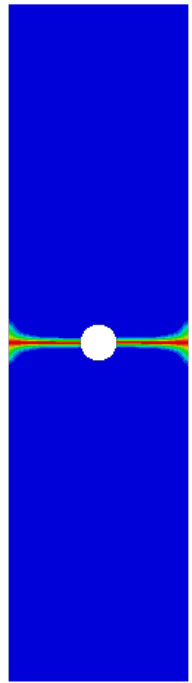

(c)

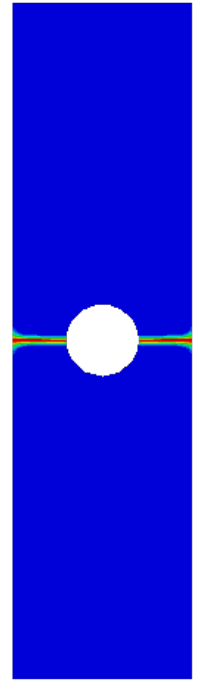

(d)
Figure 25: Open-hole tension (OHT) test on woven glass-reinforced fabric composite: Crack paths obtained from the cohesive phase-field model with parameters $p_{i}=1.0, q_{i}=0.0, L=1.2 \mathrm{~mm}$ for specimens with (a) Specimen$1[W=20 \mathrm{~mm}, D=4 \mathrm{~mm}]$ (b) Specimen-2 $[W=20 \mathrm{~mm}, D=8 \mathrm{~mm}]$ (c) Specimen-3 $[W=40 \mathrm{~mm}, D=8 \mathrm{~mm}]$ and (d) Specimen-4 $[W=40 \mathrm{~mm}$, $D=16 \mathrm{~mm}]$. The values of phase-field variable $\phi=0$ and $\phi=1$ represent intact and cracked states of the material

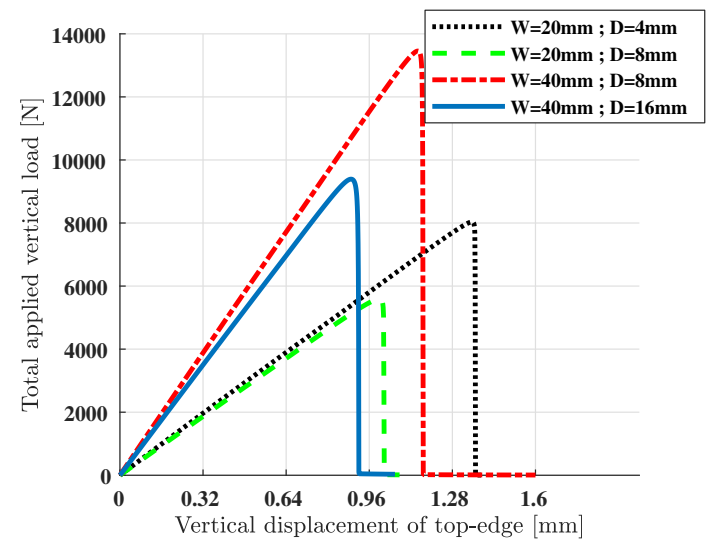

Figure 26: Open-hole tension (OHT) test on woven glass-reinforced fabric composite: Load-displacement responses for varying specimen widths $W$ and hole-diameters $D$ obtained from the cohesive phase-field model with parameters $\left[p_{i}=1.0, q_{i}=0.0, L=1.2\right]$

orientation angles, i.e., $\alpha^{\prime}=\left\{0^{\circ}, 30^{\circ}, 45^{\circ}, 60^{\circ}\right\}$. The corresponding crack-paths are shown in Fig. 30. The crack deflection angles are in good agreement with the experimental observations [103, 104] with the crack aligning consistently along the 


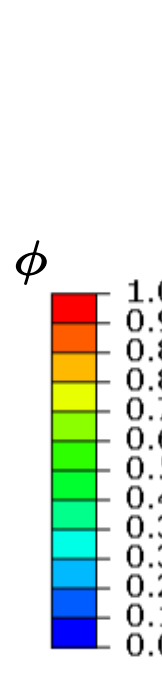

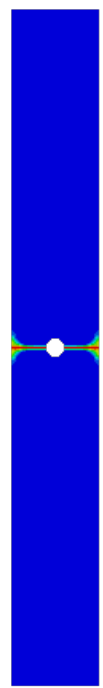

(a)

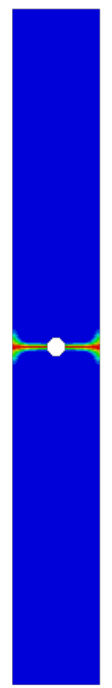

(b)

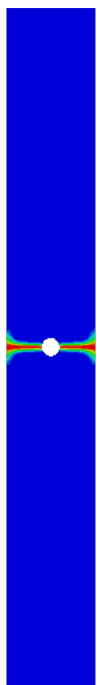

(c)
Figure 27: Open-hole tension (OHT) test on woven glass-reinforced fabric composite: Crack paths obtained for the mesh-convergence study on specimen with $W=20 \mathrm{~mm}$ and $D=4 \mathrm{~mm}$. The mesh-sizes and corresponding length-scale parameter considered are: (a) $h_{e}=0.1 \mathrm{~mm} ; L=0.6 \mathrm{~mm}$, (b) $h_{e}=0.15 \mathrm{~mm} ; L=0.9 \mathrm{~mm}$, (c) $h_{e}=0.2 \mathrm{~mm} ; L=1.2 \mathrm{~mm}$. The values of phasefield variable $\phi=0$ and $\phi=1$ represent intact and cracked states of the material

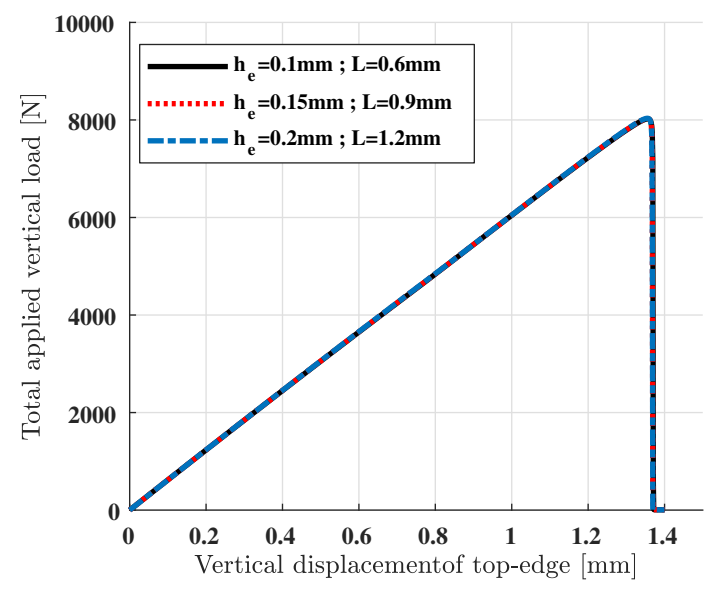

Figure 28: Open-hole tension (OHT) test on woven glass-reinforced fabric composite: Comparison of force-displacement plots obtained from the meshconvergence study on specimen with $W=20 \mathrm{~mm}$ and $D=4 \mathrm{~mm}$. The mesh-sizes and corresponding length-scale parameter considered are: (a) $h_{e}=$ $0.1 \mathrm{~mm} ; L=0.6 \mathrm{~mm}$, (b) $h_{e}=0.15 \mathrm{~mm} ; L=0.9 \mathrm{~mm}$, (c) $h_{e}=0.2 \mathrm{~mm} ; L=1.2 \mathrm{~mm}$.

fibre-directions as is usually observed in UD composites that have large difference in their fracture properties of fibre and matrix, see e.g. $[14,77]$.

The force-displacement plots for each case are shown in Fig. 31. The effective laminate strengths [Fig. 32] are compared to the experimental results provided in [103] and simulation results derived from a finite-fracture mechanics (FFM) approach [104], and brittle phase-field models [62, 76]. Although the proposed model slightly under-predicts the effective laminate strengths for the $0^{\circ}$ case by $10 \%$, the predictions for higher orientation angles $45^{\circ}$ and $60^{\circ}$ closely match the experimental results. The difference in the $0^{\circ}$ is attributed to the purely brit-

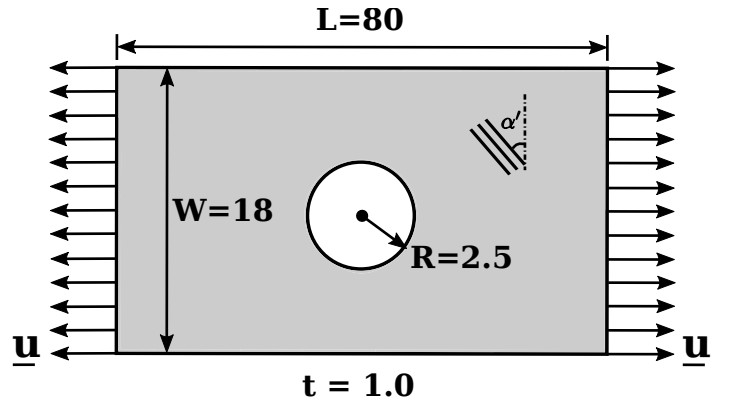

Figure 29: Open-hole tension (OHT) test on unidirectional composite laminate: Geometry and boundary conditions for quasi-UD flax fiber/epoxy matrix composite FlaxPly-UD180 with varying fibre-orientation angle $\alpha^{\prime}$ [103, 104] [All dimensions in $\mathrm{mm}$ ]

tle failure mode that this UD composite demonstrated, which cannot be accurately captured by the proposed cohesive implementation.

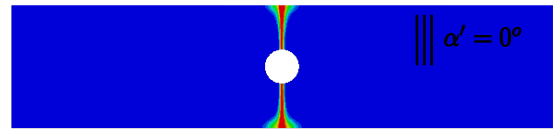

(a)
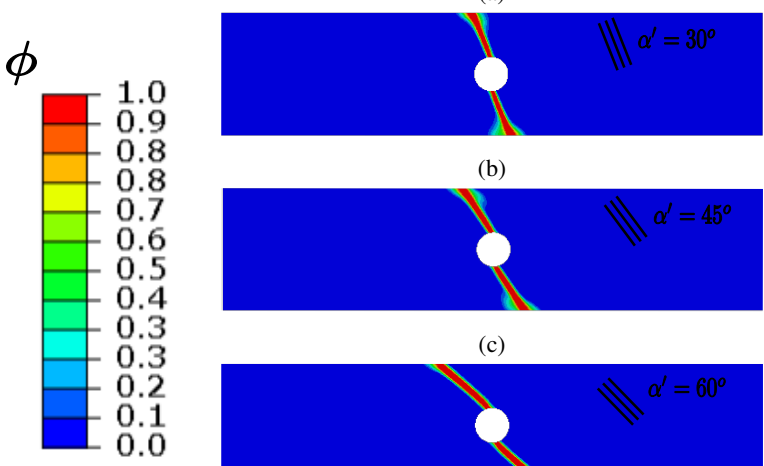

(b)

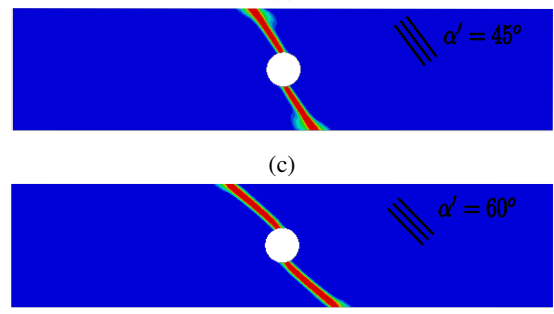

(d)

Figure 30: Open-hole tension (OHT) test on unidirectional composite laminate: Crack paths for varying fibre-orientation angles $\alpha^{\prime}=\left\{0^{\circ}, 30^{\circ}, 45^{\circ}, 60^{\circ}\right\}$. The values of phase-field variable $\phi=0$ and $\phi=1$ represent intact and cracked states of the material

\section{Conclusions}

We propose an anisotropic 3-parameter cohesive phase-field model for the simulation of quasi-brittle intralaminar damage in thin spread-tow unidirectional and woven fabric-reinforced composites. The evolving damage is represented using a single scalar phase-field variable. However, failure mode specific crack-driving force terms and corresponding degradation functions are defined hence allowing for a robust and versatile simulation tool custom fit for materials with strong anisotropies both in their elastic and fracture properties.

The stress-degradation functions are established on the basis of 3 parameters that fully describe the post-peak softening response in each failure mechanism. For the case of UD and 


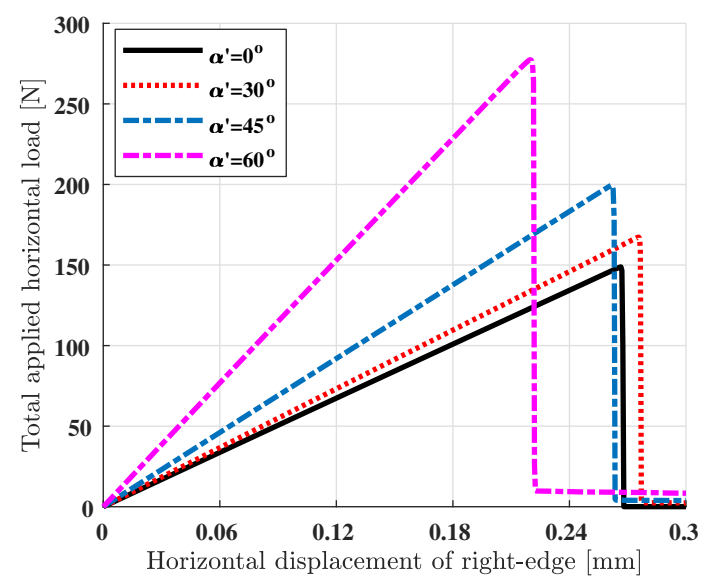

Figure 31: Open-hole tension (OHT) test on unidirectional composite laminate: Load-displacement responses for varying fibre-orientation angles $\alpha^{\prime}=$ $\left\{0^{\circ}, 30^{\circ}, 45^{\circ}, 60^{\circ}\right\}$

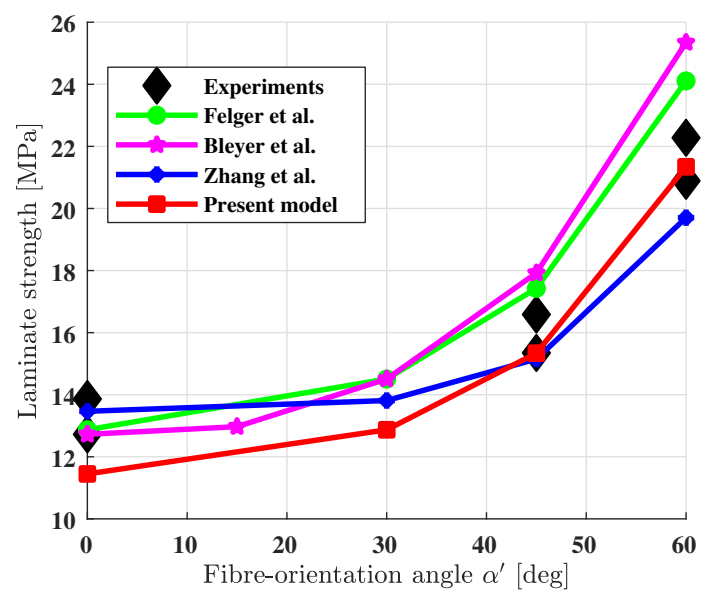

Figure 32: Open-hole tension (OHT) test on unidirectional composite laminate: Comparison of laminate strengths for different fibre orientations obtained from a) Experiments b) Finite Fracture Mechanics model in [104] c) LTD phase-field model in [62] d) Anisotropic brittle phase-field model in [76], and e) Present anisotropic cohesive phase-field model

textile composites, these can be calibrated based on three experiments, i.e., two tensile tests in two orthogonal directions and a pure shear test. A strategy to accurately calibrate the cohesive softening curve using experimental softening curves is discussed.

Modified expressions are also provided to account for the case of shear induced plastic deformations; these have been observed to be of relevance in the case of spread-tow woven fabric-reinforced composites. Within this setting, the anisotropic cohesive phase field formulations are modified to incorporate elasto-plasticity with isotropic hardening for the inplane shear behaviour.

The solution procedure for the coupled system of governing equations is solved in a staggered manner using a hybrid strategy; the crack-driving forces are evaluated based on a direct decomposition of the strain tensor but the stresses are degraded uniformly. The overall framework is implemented in the commercial software Abaqus via a user-material (UMAT) subrou- tine.

Our benchmark tests demonstrate the effectiveness of the proposed method in accurately capturing damage evolution due to diverse intra-laminar composite damage mechanisms, namely fibre fracture, fibre pull-outs/bridging, and matrix shear cracking. The proposed method is validated against two sets of experiments, i.e., CT and DENT tests performed on a Textreme ${ }^{\circledR} 80 \mathrm{~g} / \mathrm{m}^{2}$ composite. The CT tests were used to calibrate the cohesive parameters. This set of parameters accurately reproduced the DENT experimental results both in terms of the predicted crack path and the overall load deflection response of the specimen. Further validation is performed for the case of unidirectional flax ply and glass-reinforced fabric composites where the proposed model is also shown to provide accurate estimates.

Although the scope of this work is limited to simulate intralaminar fracture only, further extensions should be considered to provide a generalised framework treating both intra- and inter-laminar fracture. The extension of the proposed framework in 3D applications is feasible provided that the complex physics of through the thickness damage interactions are properly accounted for, e.g., transverse matrix shear cracks and/or the triggering of delaminations. Although in this case the definition of the corresponding crack-driving forces and the corresponding degradation functions would become more involved, no additional complexity would arise from an implementational and computational standpoint. We further aim to incorporate the proposed phase-field model within a multi-scale framework with the objective of providing accelerated simulations and hence enhancing its applicability to real-life industrial applications.

\section{Acknowledgement}

The authors would like to acknowledge the funding provided by European Union's Horizon 2020 research and innovation programme under the Marie Sklodowska-Curie SAFE-FLY project, grant agreement No. 721455 .

\section{References}

[1] B. N. Cox, G. Flanagan, Handbook of analytical methods for textile composites, 1997.

[2] E. Martín-Santos, P. Maimí, E. González, P. Cruz, A continuum constitutive model for the simulation of fabric-reinforced composites, Composite Structures 111 (2014) 122-129.

[3] K. Kawabe, T. Matsuo, Z.-i. Maekawa, New technology for opening various reinforcing fiber tows, JOURNAL-SOCIETY OF MATERIALS SCIENCE JAPAN 47 (1998) 727-734.

[4] A. Soto, E. González, P. Maimí, F. M. de la Escalera, J. S. de Aja, E. Alvarez, Low velocity impact and compression after impact simulation of thin ply laminates, Composites Part A: Applied Science and Manufacturing 109 (2018) 413-427.

[5] H. M. El-Dessouky, C. A. Lawrence, Ultra-lightweight carbon fibre/thermoplastic composite material using spread tow technology, Composites Part B: Engineering 50 (2013) 91-97.

[6] J. Tao, C. Sun, Influence of ply orientation on delamination in composite laminates, Journal of Composite Materials 32 (1998) 1933-1947.

[7] A. Arteiro, Structural mechanics of thin-ply laminated composites, Ph.D. thesis, Universidade do Porto (Portugal), 2016. 
[8] N. Khokar, F. Ohlsson, Spread tow fabrics, NetComposites https://netcomposites.com/guide/reinforcements/spread-tow-fabrics/, 2019.

[9] S. Sihn, R. Y. Kim, K. Kawabe, S. W. Tsai, Experimental studies of thin-ply laminated composites, Composites Science and Technology 67 (2007) 996-1008

[10] H. Saito, H. Takeuchi, I. Kimpara, Experimental evaluation of the damage growth restraining in 90 layer of thin-ply cfrp cross-ply laminates, Advanced Composite Materials 21 (2012) 57-66.

[11] G. Guillamet, A. Turon, J. Costa, J. Renart, Damage evolution in thin and thick-ply regions of ncf thin-ply laminates under off-axis uniaxial loading, ECCM-16TH EUROPEAN CONFERENCE ON COMPOSITE MATERIALS, Seville, Spain, 2014.

[12] A. Matzenmiller, J. Lubliner, R. Taylor, A constitutive model for anisotropic damage in fiber-composites, Mechanics of materials 20 (1995) 125-152.

[13] P. Maimí, P. P. Camanho, J. Mayugo, C. Dávila, A continuum damage model for composite laminates: Part i-constitutive model, Mechanics of Materials 39 (2007) 897-908.

[14] A. Quintanas-Corominas, J. Reinoso, E. Casoni, A. Turon, J. Mayugo, A phase field approach to simulate intralaminar and translaminar fracture in long fiber composite materials, Composite Structures 220 (2019) 899-911.

[15] S. Pinho, L. Iannucci, P. Robinson, Physically-based failure models and criteria for laminated fibre-reinforced composites with emphasis on fibre kinking: Part i: Development, Composites Part A: Applied Science and Manufacturing 37 (2006) 63-73

[16] S. Pinho, L. Iannucci, P. Robinson, Physically based failure models and criteria for laminated fibre-reinforced composites with emphasis on fibre kinking. part ii: Fe implementation, Composites Part A: Applied Science and Manufacturing 37 (2006) 766-777.

[17] P. P. Camanho, C. G. Dávila, S. T. Pinho, L. Iannucci, P. Robinson, Prediction of in situ strengths and matrix cracking in composites under transverse tension and in-plane shear, Composites Part A: Applied Science and Manufacturing 37 (2006) 165-176.

[18] M. Kachanov, Continuum model of medium with cracks, Journal of the engineering mechanics division 106 (1980) 1039-1051.

[19] Y. Liu, S. Murakami, Damage localization of conventional creep damage models and proposition of a new model for creep damage analysis, JSME International Journal Series A Solid Mechanics and Material Engineering 41 (1998) 57-65

[20] Y. N. Rabotnov, Creep problems in structural members (1969).

[21] R. Talreja, A continuum mechanics characterization of damage in composite materials, in: Proceedings of the Royal Society of London A: mathematical, physical and engineering sciences, volume 399, The Royal Society, 1985, pp. 195-216.

[22] R. Talreja, Damage mechanics of composite materials based on thermodynamics with internal variables, Durability of Polymer Based Composite Systems for Structural Applications (1990) 65-79.

[23] R. Talreja, Damage and fatigue in composites-a personal account, Composites Science and Technology 68 (2008) 2585-2591.

[24] P. Ladeveze, E. LeDantec, Damage modelling of the elementary ply for laminated composites, Composites science and technology 43 (1992) 257-267.

[25] P. Ladeveze, A damage computational method for composite structures, Computers \& Structures 44 (1992) 79-87.

[26] C. Hochard, P.-A. Aubourg, J.-P. Charles, Modelling of the mechanical behaviour of woven-fabric cfrp laminates up to failure, Composites Science and Technology 61 (2001) 221-230.

[27] A. F. Johnson, A. K. Pickett, P. Rozycki, Computational methods for predicting impact damage in composite structures, Composites Science and Technology 61 (2001) 2183-2192.

[28] C. Hochard, Y. Thollon, A generalized damage model for woven ply laminates under static and fatigue loading conditions, International Journal of Fatigue 32 (2010) 158-165.

[29] R. Böhm, M. Gude, W. Hufenbach, A phenomenologically based damage model for $2 \mathrm{~d}$ and $3 \mathrm{~d}$-textile composites with non-crimp reinforcement, Materials \& Design 32 (2011) 2532-2544.

[30] K. V. Williams, R. Vaziri, A. Poursartip, A physically based continuum damage mechanics model for thin laminated composite structures, International Journal of Solids and Structures 40 (2003) 2267-2300.
[31] L. Iannucci, R. Dechaene, M. Willows, J. Degrieck, A failure model for the analysis of thin woven glass composite structures under impact loadings, Computers \& Structures 79 (2001) 785-799.

[32] E. González, P. Maimí, E. Martín-Santos, A. Soto, P. Cruz, F. M. de la Escalera, J. S. de Aja, Simulating drop-weight impact and compression after impact tests on composite laminates using conventional shell finite elements, International Journal of Solids and Structures 144 (2018) 230 247.

[33] Z. P. Bazant, G. Pijaudier-Cabot, Nonlocal continuum damage, localization instability and convergence, Journal of applied mechanics 55 (1988) 287-293

[34] R. d. Borst, Some recent issues in computational failure mechanics, International Journal for Numerical Methods in Engineering 52 (2001) 63-95.

[35] Z. P. Bažant, B. H. Oh, Crack band theory for fracture of concrete, Matériaux et construction 16 (1983) 155-177.

[36] G. A. Francfort, J.-J. Marigo, Revisiting brittle fracture as an energy minimization problem, Journal of the Mechanics and Physics of Solids 46 (1998) 1319-1342.

[37] B. Bourdin, G. A. Francfort, J.-J. Marigo, The variational approach to fracture, Journal of elasticity 91 (2008) 5-148.

[38] H. Amor, J.-J. Marigo, C. Maurini, Regularized formulation of the variational brittle fracture with unilateral contact: Numerical experiments, Journal of the Mechanics and Physics of Solids 57 (2009) 1209-1229.

[39] C. Miehe, M. Hofacker, F. Welschinger, A phase field model for rate-independent crack propagation: Robust algorithmic implementation based on operator splits, Computer Methods in Applied Mechanics and Engineering 199 (2010) 2765-2778.

[40] M. J. Borden, C. V. Verhoosel, M. A. Scott, T. J. Hughes, C. M. Landis, A phase-field description of dynamic brittle fracture, Computer Methods in Applied Mechanics and Engineering 217 (2012) 77-95.

[41] E. Kakouris, S. P. Triantafyllou, Phase-field material point method for brittle fracture, International Journal for Numerical Methods in Engineering 112 (2017) 1750-1776

[42] E. Kakouris, S. P. Triantafyllou, Phase-field material point method for dynamic brittle fracture with isotropic and anisotropic surface energy, Computer Methods in Applied Mechanics and Engineering 357 (2019).

[43] S. Natarajan, R. K. Annabattula, E. Martínez-Pañeda, et al., Phase field modelling of crack propagation in functionally graded materials, Composites Part B: Engineering 169 (2019) 239-248.

[44] J.-Y. Wu, A unified phase-field theory for the mechanics of damage and quasi-brittle failure, Journal of the Mechanics and Physics of Solids 103 (2017) 72-99.

[45] R. J. Geelen, Y. Liu, T. Hu, M. R. Tupek, J. E. Dolbow, A phase-field formulation for dynamic cohesive fracture, Computer Methods in Applied Mechanics and Engineering 348 (2019) 680-711.

[46] M. Ambati, T. Gerasimov, L. De Lorenzis, Phase-field modeling of ductile fracture, Computational Mechanics 55 (2015) 1017-1040.

[47] C. Kuhn, T. Noll, R. Müller, On phase field modeling of ductile fracture, GAMM-Mitteilungen 39 (2016) 35-54.

[48] S. Teichtmeister, D. Kienle, F. Aldakheel, C. Miehe, Variational framework for phase field modeling of ductile fracture in porous solids at finite strains, PAMM 17 (2017) 279-280.

[49] M. Dittmann, F. Aldakheel, J. Schulte, P. Wriggers, C. Hesch, Variational phase-field formulation of non-linear ductile fracture, Computer Methods in Applied Mechanics and Engineering 342 (2018) 71-94.

[50] Y. Heider, B. Markert, A phase-field modeling approach of hydraulic fracture in saturated porous media, Mechanics Research Communications 80 (2017) 38-46.

[51] W. Ehlers, C. Luo, A phase-field approach embedded in the theory of porous media for the description of dynamic hydraulic fracturing, Computer Methods in Applied Mechanics and Engineering 315 (2017) 348368.

[52] E. Martínez-Pañeda, A. Golahmar, C. F. Niordson, A phase field formulation for hydrogen assisted cracking, Computer Methods in Applied Mechanics and Engineering 342 (2018) 742-761.

[53] M. A. Msekh, J. M. Sargado, M. Jamshidian, P. M. Areias, T. Rabczuk, Abaqus implementation of phase-field model for brittle fracture, Computational Materials Science 96 (2015) 472-484.

[54] G. Liu, Q. Li, M. A. Msekh, Z. Zuo, Abaqus implementation of monolithic and staggered schemes for quasi-static and dynamic fracture 
phase-field model, Computational Materials Science 121 (2016) 35-47.

[55] G. Molnár, A. Gravouil, 2d and 3d abaqus implementation of a robust staggered phase-field solution for modeling brittle fracture, Finite Elements in Analysis and Design 130 (2017) 27-38.

[56] U. Pillai, Y. Heider, B. Markert, A diffusive dynamic brittle fracture model for heterogeneous solids and porous materials with implementation using a user-element subroutine, Computational Materials Science 153 (2018) 36-47.

[57] C. V. Verhoosel, R. Borst, A phase-field model for cohesive fracture, International Journal for numerical methods in Engineering 96 (2013) $43-62$.

[58] J. Vignollet, S. May, R. De Borst, C. V. Verhoosel, Phase-field models for brittle and cohesive fracture, Meccanica 49 (2014) 2587-2601.

[59] S. Teichtmeister, D. Kienle, F. Aldakheel, M.-A. Keip, Phase field modeling of fracture in anisotropic brittle solids, International Journal of Non-Linear Mechanics 97 (2017) 1-21

[60] B. Li, C. Peco, D. Millán, I. Arias, M. Arroyo, Phase-field modeling and simulation of fracture in brittle materials with strongly anisotropic surface energy, International Journal for Numerical Methods in Engineering 102 (2015) 711-727.

[61] E. Kakouris, S. Triantafyllou, Material point method for crack propagation in anisotropic media: a phase field approach, Archive of Applied Mechanics 88 (2018) 287-316.

[62] J. Bleyer, R. Alessi, Phase-field modeling of anisotropic brittle fracture including several damage mechanisms, Computer Methods in Applied Mechanics and Engineering 336 (2018) 213-236.

[63] J. Reinoso, A. Arteiro, M. Paggi, P. Camanho, Strength prediction of notched thin ply laminates using finite fracture mechanics and the phase field approach, Composites Science and Technology 150 (2017) 205216.

[64] A. Dean, J. Reinoso, N. Jha, E. Mahdi, R. Rolfes, A phase field approach for ductile fracture of short fibre reinforced composites, Theoretical and Applied Fracture Mechanics (2020) 102495.

[65] S. Natarajan, R. K. Annabattula, et al., Modeling crack propagation in variable stiffness composite laminates using the phase field method, Composite Structures 209 (2019) 424-433.

[66] J. Reinoso, P. Durand, P. R. Budarapu, M. Paggi, Crack patterns in heterogenous rocks using a combined phase field-cohesive interface modeling approach: A numerical study, Energies 12 (2019) 965.

[67] T. Guillén-Hernández, I. García, J. Reinoso, M. Paggi, A micromechanical analysis of inter-fiber failure in long reinforced composites based on the phase field approach of fracture combined with the cohesive zone model, International Journal of Fracture (2019) 1-23.

[68] V. Carollo, J. Reinoso, M. Paggi, A 3d finite strain model for intralayer and interlayer crack simulation coupling the phase field approach and cohesive zone model, Composite Structures 182 (2017) 636-651.

[69] M. Paggi, J. Reinoso, Revisiting the problem of a crack impinging on an interface: a modeling framework for the interaction between the phase field approach for brittle fracture and the interface cohesive zone model, Computer Methods in Applied Mechanics and Engineering 321 (2017) $145-172$.

[70] A. Quintanas-Corominas, A. Turon, J. Reinoso, E. Casoni, M. Paggi, J. Mayugo, A phase field approach enhanced with a cohesive zone model for modeling delamination induced by matrix cracking, Computer Methods in Applied Mechanics and Engineering 358 (2020) 112618.

[71] E. Lorentz, A nonlocal damage model for plain concrete consistent with cohesive fracture, International Journal of Fracture 207 (2017) 123-159.

[72] C. G. Hoover, Z. P. Bažant, Cohesive crack, size effect, crack band and work-of-fracture models compared to comprehensive concrete fracture tests, International Journal of Fracture 187 (2014) 133-143.

[73] K. Pham, H. Amor, J.-J. Marigo, C. Maurini, Gradient damage models and their use to approximate brittle fracture, International Journal of Damage Mechanics 20 (2011) 618-652.

[74] M. Ambati, T. Gerasimov, L. De Lorenzis, A review on phase-field models of brittle fracture and a new fast hybrid formulation, Computational Mechanics 55 (2015) 383-405.

[75] T. T. Nguyen, J. Réthoré, M.-C. Baietto, Phase field modelling of anisotropic crack propagation, European Journal of Mechanics-A/Solids 65 (2017) 279-288.

[76] P. Zhang, X. Hu, T. Q. Bui, W. Yao, Phase field modeling of fracture in fiber reinforced composite laminate, International Journal of Mechanical
Sciences (2019)

[77] L. Cahill, S. Natarajan, S. P. A. Bordas, R. M. O'Higgins, C. T. McCarthy, An experimental/numerical investigation into the main driving force for crack propagation in uni-directional fibre-reinforced composite laminae, Composite Structures 107 (2014) 119-130.

[78] J.-Y. Wu, V. P. Nguyen, H. Zhou, Y. Huang, A variationally consistent phase-field anisotropic damage model for fracture, Computer Methods in Applied Mechanics and Engineering 358 (2020) 112629.

[79] A. Egger, U. Pillai, K. Agathos, E. Kakouris, E. Chatzi, I. A. Aschroft, S. P. Triantafyllou, Discrete and phase field methods for linear elastic fracture mechanics: A comparative study and state-of-the-art review, Applied Sciences 9 (2019) 2436.

[80] T. Gerasimov, L. De Lorenzis, On penalization in variational phase-field models of brittle fracture, Computer Methods in Applied Mechanics and Engineering (2019).

[81] J.-Y. Wu, V. P. Nguyen, C. T. Nguyen, D. Sutula, S. Bordas, S. Sinaie, Phase field modeling of fracture, Advances in Applied Mechancis: Multi-scale Theory and Computation 52 (2018).

[82] J.-Y. Wu, Y. Huang, V. P. Nguyen, On the bfgs monolithic algorithm for the unified phase field damage theory, Computer Methods in Applied Mechanics and Engineering 360 (2020) 112704

[83] J.-Y. Wu, Y. Huang, Comprehensive implementations of phase-field damage models in abaqus, Theoretical and Applied Fracture Mechanics 106 (2020) 102440.

[84] M. F. Wheeler, T. Wick, W. Wollner, An augmented-lagrangian method for the phase-field approach for pressurized fractures, Computer Methods in Applied Mechanics and Engineering 271 (2014) 69-85.

[85] C. Miehe, L.-M. Schaenzel, H. Ulmer, Phase field modeling of fracture in multi-physics problems. part i. balance of crack surface and failure criteria for brittle crack propagation in thermo-elastic solids, Computer Methods in Applied Mechanics and Engineering 294 (2015) 449-485.

[86] X. Zhang, S. W. Sloan, C. Vignes, D. Sheng, A modification of the phase-field model for mixed mode crack propagation in rock-like materials, Computer Methods in Applied Mechanics and Engineering 322 (2017) 123-136.

[87] V. P. Nguyen, J.-Y. Wu, Modeling dynamic fracture of solids with a phase-field regularized cohesive zone model, Computer Methods in Applied Mechanics and Engineering 340 (2018) 1000-1022

[88] J.-Y. Wu, A geometrically regularized gradient-damage model with energetic equivalence, Computer Methods in Applied Mechanics and Engineering 328 (2018) 612-637.

[89] J.-Y. Wu, Robust numerical implementation of non-standard phase-field damage models for failure in solids, Computer Methods in Applied Mechanics and Engineering 340 (2018) 767-797.

[90] J.-Y. Wu, V. P. Nguyen, A length scale insensitive phase-field damage model for brittle fracture, Journal of the Mechanics and Physics of Solids 119 (2018) 20-42.

[91] SIMULIA, ABAQUS/Standard User's Manual, Version 2017, Dassault Systèmes Simulia Corp, Providence, RI, USA, 2016.

[92] Developer Reference for Intel Math Kernel Library - Fortran, Intel Corporation, 2019. URL: https://software.intel.com/enus/mkl-developer-reference-fortran-intel-mkl-pardisoparallel-direct-sparse-solver-interface.

[93] A. Quintanas-Corominas, P. Maimí, E. Casoni, A. Turon, J. A. Mayugo, G. Guillamet, M. Vázquez, A 3d transversally isotropic constitutive model for advanced composites implemented in a high performance computing code, European Journal of Mechanics-A/Solids 71 (2018) $278-291$

[94] DAMTEXproject, Report with a summary of the tests and the data reduction methods applied for fibre fracture toughness characterization, under tension and compression loading (TeXtreme $80 \mathrm{gsm}$ material), Technical Report E2.1 (2013-DAM-01-IT06), Aernnova Engineering Division S.A. (Spain) ; AMADE Polytechnic school, University of Girona (Spain), 2014.

[95] OxeonAB, TeXtreme, http://www.textreme.com/, 2003.

[96] DAMTEXproject, Report of FE models for the prediction of impact and compression after impact tests, Technical Report E4.2, E4.3, E5.1 (2013-DAM-01-IT10), Aernnova Engineering Division S.A. (Spain) ; AMADE Polytechnic school, University of Girona (Spain), 2014.

[97] E. Gonzalez, A. Soto, Material characterization tests (TeXtreme 80 gsm material), Technical Report DAMTEXproject 2013-DAM-01- 
IT04-SummaryMaterialProperties, Aernnova Engineering Division S.A (Spain) ; AMADE Polytechnic school, University of Girona (Spain), 2015.

[98] A. Wagih, P. Maimí, E. González, N. Blanco, J. S. de Aja, F. De La Escalera, R. Olsson, E. Alvarez, Damage sequence in thin-ply composite laminates under out-of-plane loading, Composites Part A: Applied Science and Manufacturing 87 (2016) 66-77.

[99] Z. P. Bazant, Fracture and size effect in concrete and other quasibrittle materials, Routledge, 2019.

[100] J.-K. Kim, D.-S. Kim, N. Takeda, Notched strength and fracture criterion in fabric composite plates containing a circular hole, Journal of composite materials 29 (1995) 982-998.

[101] H. Ahmad, Finite element-based strength prediction for notched and mechanically fastened woven fabric composites, Ph.D. thesis, University of Surrey, 2012.

[102] A. Arteiro, G. Catalanotti, J. Xavier, P. Camanho, Notched response of non-crimp fabric thin-ply laminates, Composites Science and Technology 79 (2013) 97-114.

[103] J. Modniks, E. Spārninšs, J. Andersons, W. Becker, Analysis of the effect of a stress raiser on the strength of a ud flax/epoxy composite in off-axis tension, Journal of Composite Materials 49 (2015) 1071-1080.

[104] J. Felger, N. Stein, W. Becker, Mixed-mode fracture in open-hole composite plates of finite-width: An asymptotic coupled stress and energy approach, International Journal of Solids and Structures 122 (2017) 1424.

[105] FLAXPREG, https://eco-technilin.com/en/\#!products/vstc3=flaxpreg, 2012. 